Research Article

\title{
Symplectic Synchronization of Lorenz-Stenflo System with Uncertain Chaotic Parameters via Adaptive Control
}

\author{
Cheng-Hsiung Yang \\ Graduate Institute of Automation and Control, National Taiwan University of Science and Technology, 43 Section 4, \\ Keelung Road, Taipei 106, Taiwan \\ Correspondence should be addressed to Cheng-Hsiung Yang; chyang123123@mail.ntust.edu.tw
}

Received 24 October 2012; Accepted 30 December 2012

Academic Editor: Chuandong Li

Copyright (c) 2013 Cheng-Hsiung Yang. This is an open access article distributed under the Creative Commons Attribution License, which permits unrestricted use, distribution, and reproduction in any medium, provided the original work is properly cited.

A new symplectic chaos synchronization of chaotic systems with uncertain chaotic parameters is studied. The traditional chaos synchronizations are special cases of the symplectic chaos synchronization. A sufficient condition is given for the asymptotical stability of the null solution of error dynamics and a parameter difference. The symplectic chaos synchronization with uncertain chaotic parameters may be applied to the design of secure communication systems. Finally, numerical results are studied for symplectic chaos synchronized from two identical Lorenz-Stenflo systems in three different cases.

\section{Introduction}

Chaos has been detected in a large number of nonlinear dynamic systems of physical characteristics. In addition to the control and stabilization of chaos, chaos synchronization systems are a fascinating concept which has received considerable interest among nonlinear scientists in recent times. However, chaos is desirable in some systems, such as convective heat transfer, liquid mixing, encryption, power converters, secure communications, biological systems and chemical reactions. There are a chaotic master system (or driver) and either an identical or a different slave system (or responser). Our goal is the synchronization of the chaotic master system and the chaotic slave system by coupling or by other methods. In practice, some or all of the parameters of chaotic system parameters are uncertain. A lot of works have been preceded to solve this problem using adaptive control concept.

Among many kinds of chaos synchronization, generalized chaos synchronization is investigated [1-6]. There exists a functional relationship between the states of the master system and those of the slave system. The symplectic chaos synchronization concept [7]:

$$
y=H(t, x, y)+F(t)
$$

is studied, where $x, y$ are the state vectors of the master system and of the slave system, respectively, $F(t)$ is a given function of time in different form. The $F(t)$ may be a regular function or a chaotic function. When $H(t, x, y)+F(t)=$ $x$ and $H(t, x, y)=x$ of (1) reduces to the traditional generalized chaos synchronization and the traditional chaos synchronization given in [8-10], respectively.

As numerical examples, in 1996, Stenflo originally used a four-dimensional autonomous chaotic system to describe the low-frequency short-wavelength gravity wave disturbance in the atmosphere [11]. This system is similar to the celebrated Lorenz equation but more complex than it due to the introduction of a new feedback control and a new state variable and thus is called a Lorenz-Stenflo system after the names of Lorenz and Stenflo [12]. The nonlinear dynamical behaviors of the Lorenz-Stenflo system have been investigated in [13$16]$.

This paper is organized as follows. In Section 2, by the Lyapunov asymptotic stability theorem, the symplectic chaos synchronization with uncertain chaotic parameters by adaptive control scheme is given. In Section 3, various adaptive controllers and update laws are designed for the symplectic chaos synchronization of the identical LorenzStenflo systems. Numerical simulations are also given in Section 3. Finally, some concluding remarks are given in Section 4. 


\section{Symplectic Chaos Synchronization with Chaotic Parameters by Adaptive Control Scheme}

There are two identical nonlinear dynamical systems, and the partner $A$ controls the partner $B$. The partner $A$ is given by

$$
\dot{x}=f(t, x, A(t)) \text {, }
$$

where $x=\left[x_{1}, x_{2}, \ldots, x_{n}\right]^{T} \in R^{n}$ is a state vector, $A(t)=$ $\left[A_{1}(t), A_{2}(t), \ldots, A_{m}(t)\right]^{T} \in R^{m}$ is a vector of uncertain coefficients in $f$, and $f$ is a vector function.

The partner $B$ is given by

$$
\dot{y}=f(t, y, \widehat{A}(t)),
$$

where $y=\left[y_{1}, y_{2}, \ldots, y_{n}\right]^{T} \in R^{n}$ is a state vector, $\widehat{A}(t)=$ $\left[\widehat{A}_{1}(t), \widehat{A}_{2}(t), \ldots, \widehat{A}_{m}(t)\right]^{T} \in R^{m}$ is an estimated vector of uncertain coefficients in $f$.

So a controller $u(t)$ is added on partner $B$, and the partner $B$ becomes

$$
\dot{y}=f(t, y, \widehat{A}(t))+u(t)
$$

where $u(t)=\left[u_{1}(t), u_{2}(t), \ldots, u_{n}(t)\right]^{T} \in R^{n}$ is the control vector function.

Our goal is to design the controller $u(t)$ so that the state vector $y$ of the partner $B$ asymptotically approaches $H(t, x, y)+F(t)$, a given function $H(t, x, y)$ plus a given vector function $F(t)=\left[F_{1}(t), F_{2}(t), \ldots, F_{n}(t)\right]^{T}$ which is the regular function or the chaotic function.

To define error vector $e(t)=\left[e_{1}, e_{2}, \ldots, e_{n}\right]^{T}$ :

$$
\begin{gathered}
e=H(t, x, y)-y+F(t), \\
\lim _{t \rightarrow \infty} e=0
\end{gathered}
$$

is demanded.

From (4), it is obtained that

$$
\dot{e}=\frac{\partial H}{\partial t}+\nabla H^{T} \dot{\Psi}-\dot{y}+\dot{F}(t)
$$

where $\dot{\Psi}^{T}=\left[\begin{array}{ll}\dot{x} & \dot{y}\end{array}\right]$.

By (2), (3a), and (3b), (6) can be rewritten as

$$
\begin{aligned}
\dot{e}= & \frac{\partial H}{\partial t}+\frac{\partial H}{\partial x} f(t, x, A(t))+\frac{\partial H}{\partial y} f(t, y, \widehat{A}(t)) \\
& -f(t, y, \widehat{A}(t))-u(t)+\dot{F}(t) .
\end{aligned}
$$
sen:

A positive definite Lyapunov function $V(e, \widetilde{A}(t))$ is cho-

$$
V(e, \widetilde{A}(t))=\frac{1}{2} e^{T} e+\frac{1}{2} \widetilde{A}^{T}(t) \widetilde{A}(t),
$$

where $\widetilde{A}(t)=A(t)-\widehat{A}(t)$.
Its derivative along any solution of (7) is

$$
\begin{aligned}
\dot{V}(e, \widetilde{A}(t))= & e^{T}\left\{\frac{\partial H}{\partial t}+\frac{\partial H}{\partial x} f(t, x, A(t))+\frac{\partial H}{\partial y} f(t, y, \widehat{A}(t))\right. \\
& -f(t, y, \widehat{A}(t))+\dot{F}(t)-u(t)\} \\
& +\widetilde{A}^{T}(t) \dot{\widetilde{A}}(t) .
\end{aligned}
$$

In (9), the $u(t)$ and $\tilde{\widetilde{A}}(t)$ are designed so that $\dot{V}=e^{T} C_{n \times n} e+$ $\widetilde{A}^{T}(t) D_{m \times m} \widetilde{A}(t)$ where $C_{n \times n}$ and $D_{m \times m}$ are two diagonal negative definite matrices. The $\dot{V}$ is a negative definite function of $e$ and $\widetilde{A}(t)$. By Lyapunov theorem of asymptotical stability

$$
\lim _{t \rightarrow \infty} e=0, \quad \lim _{t \rightarrow \infty} \widetilde{A}(t)=0 .
$$

The symplectic chaos synchronization with uncertain chaotic parameters is obtained [3-7, 11, 17-23].

\section{Numerical Results for the Symplectic \\ Chaos Synchronization of Lorenz-Stenflo System with Uncertain Chaotic Parameters via Adaptive Control}

The master Lorenz-Stenflo system can be described as [11]

$$
\begin{gathered}
\dot{x}_{1}=a\left(x_{2}-x_{1}\right)+r x_{4}, \\
\dot{x}_{2}=d x_{1}-x_{2}-x_{1} x_{3}, \\
\dot{x}_{3}=-b x_{3}+x_{1} x_{2}, \\
\dot{x}_{4}=-x_{4}-c x_{1} .
\end{gathered}
$$

And the slave Lorenz-Stenflo system can be described as

$$
\begin{gathered}
\dot{y}_{1}=\widehat{a}\left(y_{2}-y_{1}\right)+\widehat{r} y_{4}, \\
\dot{y}_{2}=\widehat{d} y_{1}-y_{2}-y_{1} y_{3}, \\
\dot{y}_{3}=-\widehat{b} y_{3}+y_{1} y_{2}, \\
\dot{y}_{4}=-y_{4}-\widehat{c} y_{1},
\end{gathered}
$$

where $a, b, c, d$, and $r$ are called the first Prandtl number, geometric parameter, second Prandtl number, Rayleigh number, and rotation number, respectively. The parameters of LorenzStenflo system are chosen as $a=11, b=2.9, c=5, d=23$, and $r=1.9$.

The controllers, $u_{1}, u_{2}, u_{3}$, and $u_{4}$, are added to the four equations of (12), respectively:

$$
\begin{aligned}
& \dot{y}_{1}=\widehat{a}\left(y_{2}-y_{1}\right)+\widehat{r} y_{4}+u_{1}, \\
& \dot{y}_{2}=\widehat{d} y_{1}-y_{2}-y_{1} y_{3}+u_{2},
\end{aligned}
$$




$$
\begin{gathered}
\dot{y}_{3}=-\widehat{b} y_{3}+y_{1} y_{2}+u_{3}, \\
\dot{y}_{4}=-y_{4}-\widehat{c} y_{1}+u_{4} .
\end{gathered}
$$

The initial values of the states of the master system and of the slave system are taken as $x_{1}(0)=10, x_{2}(0)=-13$, $x_{3}(0)=12, x_{4}(0)=-5, y_{1}(0)=-3, y_{2}(0)=-4, y_{3}(0)=3$, and $y_{4}(0)=-5$.

Case 1. A time delay symplectic synchronization.

We take $F_{1}(t)=x_{1}(t-T), F_{2}(t)=x_{2}(t-T), F_{3}(t)=$ $x_{3}(t-T)$, and $F_{4}(t)=x_{4}(t-T)$. They are chaotic functions of time, where time delay $T=0.5 \mathrm{sec}$. The $H_{i}(x, y, t)=\left(x_{i}^{2}+\right.$ $\left.y_{i}\right)(\sin w t+3)(i=1,2,3,4)$ are given. By $(5)$ we have

$$
\begin{array}{r}
\lim _{t \rightarrow \infty} e_{i}=\lim _{t \rightarrow \infty}\left(\left(x_{i}^{2}+y_{i}\right)(\sin \omega t+3)-y_{i}+x_{i}(t-T)\right)=0 \\
i=1,2,3,4 .
\end{array}
$$

From (14) we have

$$
\begin{aligned}
\dot{e}_{i}= & \left(2 x_{i} \dot{x}_{i}+\dot{y}_{i}\right)(\sin \omega t+3)+\omega \cos \omega t\left(x_{i}^{2}+y_{i}\right) \\
& -\dot{y}_{i}+\dot{x}_{i}(t-T), \quad i=1,2,3,4
\end{aligned}
$$

where $\omega=19$.

Equation (15) can be expressed as

$$
\begin{aligned}
\dot{e}_{1}= & {\left[2 x_{1}\left(a\left(x_{2}-x_{1}\right)+r x_{4}\right)+\left(\widehat{a}\left(y_{2}-y_{1}\right)+\widehat{r} y_{4}\right)\right] } \\
& \times(\sin \omega t+3)+\omega \cos \omega t\left(x_{1}^{2}+y_{1}\right)-\widehat{a}\left(y_{2}-y_{1}\right) \\
& -\widehat{r} y_{4}-u_{1}+a\left(x_{2}(t-T)-x_{1}(t-T)\right)+r x_{4}(t-T), \\
\dot{e}_{2}= & {\left[2 x_{2}\left(d x_{1}-x_{2}-x_{1} x_{3}\right)+\left(\widehat{d} y_{1}-y_{2}-y_{1} y_{3}\right)\right] } \\
& \times(\sin \omega t+3)+\omega \cos \omega t\left(x_{2}^{2}+y_{2}\right) \\
& -\widehat{d}_{1}+y_{2}+y_{1} y_{3}+u_{2} \\
& +d x_{1}(t-T)-x_{2}(t-T)-x_{1}(t-T) x_{3}(t-T), \\
\dot{e}_{3}= & {\left[2 x_{3}\left(-b x_{3}+x_{1} x_{2}\right)-\widehat{b} y_{3}+y_{1} y_{2}\right](\sin \omega t+3) } \\
& +\omega \cos \omega t\left(x_{3}^{2}+y_{3}\right)+\widehat{b} y_{3}-y_{1} y_{2}-u_{3} \\
& -b x_{3}(t-T)+x_{1}(t-T) x_{2}(t-T),
\end{aligned}
$$

$$
\begin{aligned}
\dot{e}_{4}= & {\left[2 x_{4}\left(-x_{4}-c x_{1}\right)-y_{4}-\widehat{c} y_{1}\right](\sin \omega t+3) } \\
& +\omega \cos \omega t\left(x_{4}^{2}+y_{4}\right)+y_{4}+\widehat{c} y_{1}-u_{4} \\
& -x_{4}(t-T)-c x_{1}(t-T),
\end{aligned}
$$

where

$$
\begin{aligned}
& e_{1}=\left(x_{1}^{2}+y_{1}\right)(\sin \omega t+3)-y_{1}+x_{1}(t-T), \\
& e_{2}=\left(x_{2}^{2}+y_{2}\right)(\sin \omega t+3)-y_{2}+x_{2}(t-T), \\
& e_{3}=\left(x_{3}^{2}+y_{3}\right)(\sin \omega t+3)-y_{3}+x_{3}(t-T), \\
& e_{4}=\left(x_{4}^{2}+y_{4}\right)(\sin \omega t+3)-y_{4}+x_{4}(t-T) .
\end{aligned}
$$

Choose a positive definite Lyapunov function:

$$
\begin{aligned}
& V\left(e_{1}, e_{2}, e_{3}, e_{4}, \tilde{a}, \tilde{b}, \tilde{c}, \tilde{d}, \tilde{r}\right) \\
& \quad=\frac{1}{2}\left(e_{1}^{2}+e_{2}^{2}+e_{3}^{2}+e_{4}^{2}+\widetilde{a}^{2}+\widetilde{b}^{2}+\widetilde{c}^{2}+\widetilde{d}^{2}+\widetilde{r}^{2}\right),
\end{aligned}
$$

where $\tilde{a}=a-\widehat{a}, \tilde{b}=b-\widehat{b}, \widetilde{c}=c-\widehat{c}, \tilde{d}=d-\widehat{d}, \tilde{r}=r-\widehat{r}$, and $\widehat{a}, \widehat{b}, \widehat{c}, \widehat{d}$, and $\widehat{r}$ are estimates of uncertain parameters $\mathrm{a}, \mathrm{b}, \mathrm{c}$, $\mathrm{d}$, and $\mathrm{r}$, respectively.

Its time derivative along any solution of (16) is

$$
\begin{aligned}
& \dot{V}=e_{1}\{[\left.2 x_{1}\left(a\left(x_{2}-x_{1}\right)+r x_{4}\right)+\left(\widehat{a}\left(y_{2}-y_{1}\right)+\widehat{r} y_{4}\right)\right] \\
& \times(\sin \omega t+3)+\omega \cos \omega t\left(x_{1}^{2}+y_{1}\right)-\widehat{a}\left(y_{2}-y_{1}\right) \\
&-\left.\widehat{r} y_{4}-u_{1}+a\left(x_{2}(t-T)-x_{1}(t-T)\right)+r x_{4}(t-T)\right\} \\
&+e_{2}\left\{\left[2 x_{2}\left(d x_{1}-x_{2}-x_{1} x_{3}\right)+\left(\widehat{d} y_{1}-y_{2}-y_{1} y_{3}\right)\right]\right. \\
& \times(\sin \omega t+3)+\omega \cos \omega t\left(x_{2}^{2}+y_{2}\right)-\widehat{d} y_{1}+y_{2} \\
&+y_{1} y_{3}-u_{2}+d x_{1}(t-T)-x_{2}(t-T) \\
&\left.-x_{1}(t-T) x_{3}(t-T)\right\} \\
&+e_{3}\left\{\left[2 x_{3}\left(-b x_{3}+x_{1} x_{2}\right)-\widehat{b} y_{3}+y_{1} y_{2}\right]\right. \\
& \times(\sin \omega t+3)+\omega \cos \omega t\left(x_{3}^{2}+y_{3}\right)+\widehat{b} y_{3} \\
&-y_{1} y_{2}-u_{3}-b x_{3}(t-T) \\
&\left.+x_{1}(t-T) x_{2}(t-T)\right\}
\end{aligned}
$$




$$
\begin{aligned}
+e_{4}\{ & {\left[2 x_{4}\left(-x_{4}-c x_{1}\right)-y_{4}-\widehat{c} y_{1}\right](\sin \omega t+3) } \\
+ & \omega \cos \omega t\left(x_{4}^{2}+y_{4}\right)+y_{4}+\widehat{c} y_{1}-u_{4} \\
& \left.-x_{4}(t-T)-c x_{1}(t-T)\right\}+\tilde{a} \dot{\tilde{a}}+\tilde{b} \tilde{b} \\
+\dot{\vec{c}}+\tilde{d} & \dot{d} \tilde{d}+\dot{r} \dot{r}
\end{aligned}
$$

The adaptive controllers are chosen as

$$
\begin{aligned}
u_{1}= & {\left[2 x_{1}\left(a\left(x_{2}-x_{1}\right)+r x_{4}\right)+\left(a\left(y_{2}-y_{1}\right)+r y_{4}\right)\right] } \\
& \times(\sin \omega t+3)+\omega \cos \omega t\left(x_{1}^{2}+y_{1}\right)-a\left(y_{2}-y_{1}\right) \\
& -r y_{4}+a\left(x_{2}(t-T)-x_{1}(t-T)\right)+r x_{4}(t-T)+e_{1}, \\
u_{2}= & {\left[2 x_{2}\left(d x_{1}-x_{2}-x_{1} x_{3}\right)+\left(d y_{1}-y_{2}-y_{1} y_{3}\right)\right] } \\
& \times(\sin \omega t+3)+\omega \cos \omega t\left(x_{2}^{2}+y_{2}\right) \\
& -d y_{1}+y_{2}+y_{1} y_{3}+d x_{1}(t-T) \\
& -x_{2}(t-T)-x_{1}(t-T) x_{3}(t-T)+e_{2}, \\
u_{3}= & {\left[2 x_{3}\left(-b x_{3}+x_{1} x_{2}\right)-b y_{3}+y_{1} y_{2}\right](\sin \omega t+3) } \\
& +\omega \cos \omega t\left(x_{3}^{2}+y_{3}\right)+b y_{3}-y_{1} y_{2} \\
& -b x_{3}(t-T)+x_{1}(t-T) x_{2}(t-T)+e_{3}, \\
u_{4}= & {\left[2 x_{4}\left(-x_{4}-c x_{1}\right)-y_{4}-c y_{1}\right](\sin \omega t+3) } \\
& +\omega \cos \omega t\left(x_{4}^{2}+y_{4}\right)+y_{4}+c y_{1} \\
& -x_{4}(t-T)-c x_{1}(t-T)+e_{4},
\end{aligned}
$$

and the update laws are chosen as

$$
\begin{aligned}
\dot{\tilde{a}}=-\dot{\widehat{a}} & =2 x_{1}\left(y_{2}-y_{1}\right)(\sin \omega t+3) e_{1}+\left(y_{2}-y_{1}\right) e_{1}-\tilde{a}, \\
\dot{\tilde{b}} & =-\dot{\widehat{b}}=-2 x_{3} y_{3} e_{3}(\sin \omega t+3)-y_{3} e_{3}-\tilde{b}, \\
\dot{\widetilde{c}} & =-\dot{\widehat{c}}=-2 x_{4} y_{1} e_{4}(\sin \omega t+3)-y_{1} e_{4}-\tilde{c}, \\
\dot{\tilde{d}} & =-\dot{\widehat{d}}=2 x_{2} y_{1} e_{2}(\sin \omega t+3)+y_{1} e_{2}-\tilde{d}, \\
\dot{\widetilde{r}} & =-\dot{\widehat{r}}=2 x_{1} y_{4} e_{1}(\sin \omega t+3)+y_{4} e_{1}-\tilde{r} .
\end{aligned}
$$

The initial values of estimate for uncertain parameters are $\widehat{a}(0)=\widehat{b}(0)=\widehat{c}(0)=\widehat{d}(0)=\widehat{r}(0)=0$.

Equation (19) becomes

$$
\dot{V}=-\left(e_{1}^{2}+e_{2}^{2}+e_{3}^{2}+e_{4}^{2}+\widetilde{a}^{2}+\widetilde{b}^{2}+\widetilde{c}^{2}+\widetilde{d}^{2}+\widetilde{r}^{2}\right)<0
$$

which is negative definite. The Lyapunov asymptotical stability theorem is satisfied. The time delay symplectic synchronization of the identical Lorenz-Stenflo systems is achieved.
The numerical results of the phase portrait of master system, chaotic system (3), the time series of states, state errors, and parameter differences are shown in Figures 1, 2, and 3, respectively. The symplectic chaos synchronization is accomplished using adaptive control method.

Case 2. A time delay symplectic synchronization with uncertain chaotic parameters.

The Lorenz-Stenflo master system with uncertain chaotic parameters is

$$
\begin{gathered}
\dot{x}_{1}=A(t)\left(x_{2}-x_{1}\right)+R(t) x_{4}, \\
\dot{x}_{2}=D(t) x_{1}-x_{2}-x_{1} x_{3}, \\
\dot{x}_{3}=-B(t) x_{3}+x_{1} x_{2}, \\
\dot{x}_{4}=-x_{4}-C(t) x_{1},
\end{gathered}
$$

where $A(t), B(t), C(t), D(t)$, and $R(t)$ are uncertain chaotic parameters. In simulation, we take

$$
\begin{gathered}
A(t)=a\left(1+k_{1} z_{1}\right), \quad B(t)=b\left(1+k_{2} z_{3}\right), \\
C(t)=c\left(1+k_{3} z_{4}\right), \quad D(t)=d\left(1+k_{4} z_{2}\right), \\
R(t)=r\left(1+k_{5} z_{1}\right),
\end{gathered}
$$

where $k_{1}, k_{2}, k_{3}, k_{4}$, and $k_{5}$ are positive constants. Chosen are $k_{1}=0.07, k_{2}=0.08, k_{3}=0.06, k_{4}=0.07$, and $k_{5}=0.08$. The system (23) is chaotic dynamic motion, shown in Figure 4.

The $F(t)$ is chaotic system, and the chaotic signal of goal system can be described as

$$
\begin{gathered}
\dot{z}_{1}=a\left(z_{2}-z_{1}\right)+r z_{4}, \\
\dot{z}_{2}=d z_{1}-z_{2}-z_{1} z_{3}, \\
\dot{z}_{3}=-b z_{3}+z_{1} z_{2}, \\
\dot{z}_{4}=-z_{4}-c z_{1}
\end{gathered}
$$

where initial conditions of the chaotic signal of system are $z_{1}(0)=2, z_{2}(0)=5, z_{3}(0)=-4$, and $z_{4}(0)=-6$. The $H_{i}(x, y, t)=\left(x_{i}^{2}+y_{i}\right)(\sin w t+3)(i=1,2,3,4)$ are given. By (5) we have

$$
\lim _{t \rightarrow \infty} e_{i}=\lim _{t \rightarrow \infty}\left(\left(x_{i}^{2}+y_{i}\right)(\sin \omega t+3)-y_{i}+x_{i}(t-T)\right)=0,
$$

$$
i=1,2,3,4 \text {. }
$$

From (26) we have

$$
\begin{aligned}
\dot{e}_{i}= & \left(2 x_{i} \dot{x}_{i}+\dot{y}_{i}\right)(\sin \omega t+3)+\omega \cos \omega t\left(x_{i}^{2}+y_{i}\right) \\
& -\dot{y}_{i}+\dot{x}_{i}(t-T), \quad i=1,2,3,4 .
\end{aligned}
$$




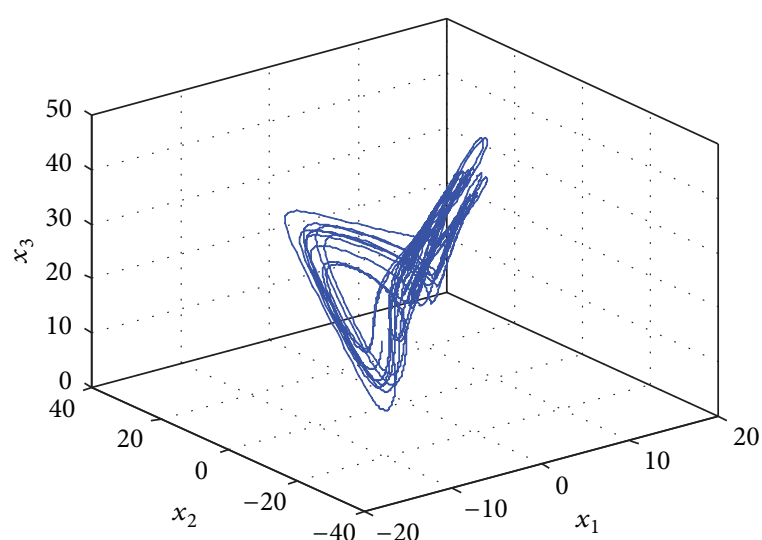

(a)

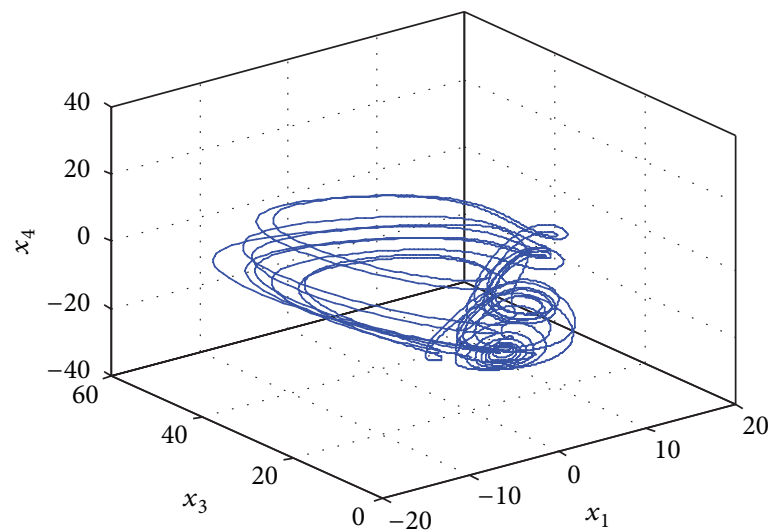

(c)

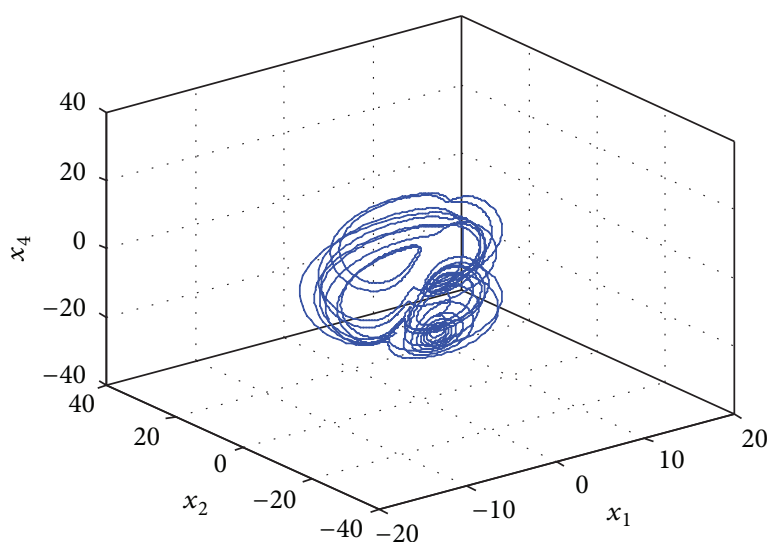

(b)

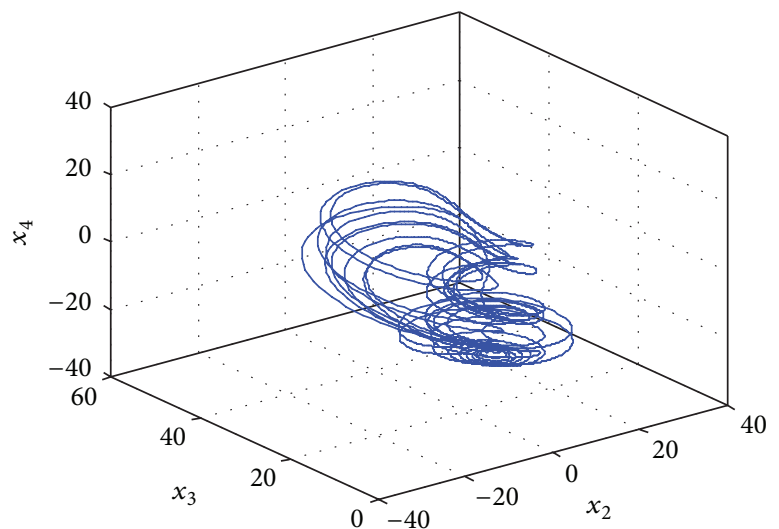

(d)

FIGURE 1: Projections of phase portrait for master system (11).

Equation (27) can be expressed as

$$
\begin{aligned}
\dot{e}_{1}= & {\left[2 x_{1}\left(A(t)\left(x_{2}-x_{1}\right)+R(t) x_{4}\right)+\left(\widehat{a}\left(y_{2}-y_{1}\right)+\widehat{r} y_{4}\right)\right] } \\
& \times(\sin \omega t+3)+\omega \cos \omega t\left(x_{1}^{2}+y_{1}\right)-\widehat{a}\left(y_{2}-y_{1}\right) \\
& -\widehat{r} y_{4}-u_{1}+A(t)\left(x_{2}(t-T)-x_{1}(t-T)\right) \\
& +R(t) x_{4}(t-T), \\
\dot{e}_{2}= & {\left[2 x_{2}\left(D(t) x_{1}-x_{2}-x_{1} x_{3}\right)+\left(\widehat{d} y_{1}-y_{2}-y_{1} y_{3}\right)\right] } \\
& \times(\sin \omega t+3)+\omega \cos \omega t\left(x_{2}^{2}+y_{2}\right) \\
& -\widehat{d} y_{1}+y_{2}+y_{1} y_{3}+u_{2}+D(t) x_{1}(t-T) \\
& -x_{2}(t-T)-x_{1}(t-T) x_{3}(t-T), \\
\dot{e}_{3}= & {\left[2 x_{3}\left(-B(t) x_{3}+x_{1} x_{2}\right)-\widehat{b} y_{3}+y_{1} y_{2}\right](\sin \omega t+3) } \\
& +\omega \cos \omega t\left(x_{3}^{2}+y_{3}\right)+\widehat{b} y_{3}-y_{1} y_{2}-u_{3} \\
& -B(t) x_{3}(t-T)+x_{1}(t-T) x_{2}(t-T), \\
\dot{e}_{4}= & {\left[2 x_{4}\left(-x_{4}-C(t) x_{1}\right)-y_{4}-\widehat{c} y_{1}\right](\sin \omega t+3) } \\
& +\omega \cos \omega t\left(x_{4}^{2}+y_{4}\right)+y_{4}+\widehat{c} y_{1}-u_{4} \\
& -x_{4}(t-T)-C(t) x_{1}(t-T),
\end{aligned}
$$

where

$$
\begin{aligned}
& e_{1}=\left(x_{1}^{2}+y_{1}\right)(\sin \omega t+3)-y_{1}+x_{1}(t-T), \\
& e_{2}=\left(x_{2}^{2}+y_{2}\right)(\sin \omega t+3)-y_{2}+x_{2}(t-T), \\
& e_{3}=\left(x_{3}^{2}+y_{3}\right)(\sin \omega t+3)-y_{3}+x_{3}(t-T), \\
& e_{4}=\left(x_{4}^{2}+y_{4}\right)(\sin \omega t+3)-y_{4}+x_{4}(t-T) .
\end{aligned}
$$

Choose a positive definite Lyapunov function:

$$
\begin{aligned}
& V\left(e_{1}, e_{2}, e_{3}, e_{4}, \tilde{a}, \tilde{b}, \tilde{c}, \tilde{d}, \tilde{r}\right) \\
& \quad=\frac{1}{2}\left(e_{1}^{2}+e_{2}^{2}+e_{3}^{2}+e_{4}^{2}+\widetilde{a}^{2}+\widetilde{b}^{2}+\widetilde{c}^{2}+\widetilde{d}^{2}+\widetilde{r}^{2}\right),
\end{aligned}
$$

where $\tilde{a}=A(t)-\widehat{a}, \tilde{b}=B(t)-\widehat{b}, \tilde{c}=C(t)-\widehat{c}, \tilde{d}=D(t)-\widehat{d}$, $\widetilde{r}=R(t)-\widehat{r}$, and $\widehat{a}, \widehat{b}, \widehat{c}, \widehat{d}$, and $\widehat{r}$ are estimates of uncertain parameters $A(t), B(t), C(t), D(t)$, and $R(t)$, respectively. 


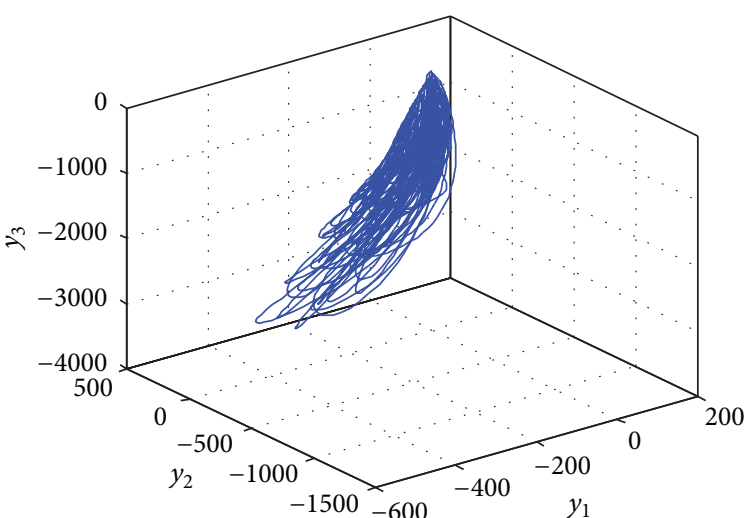

(a)

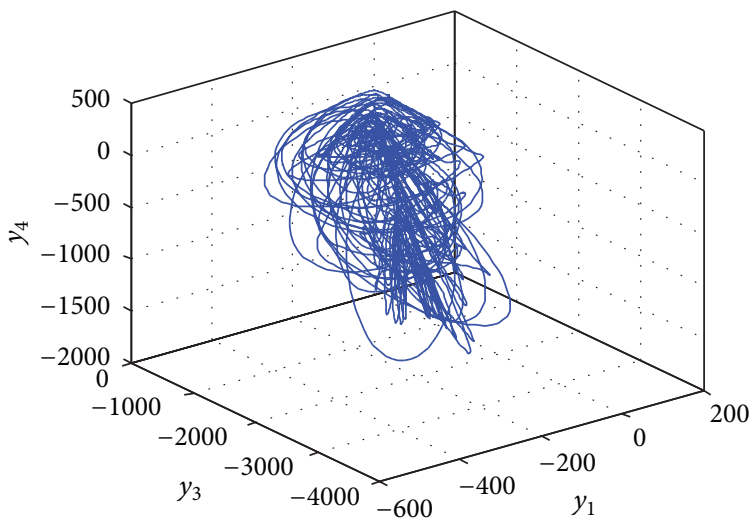

(c)

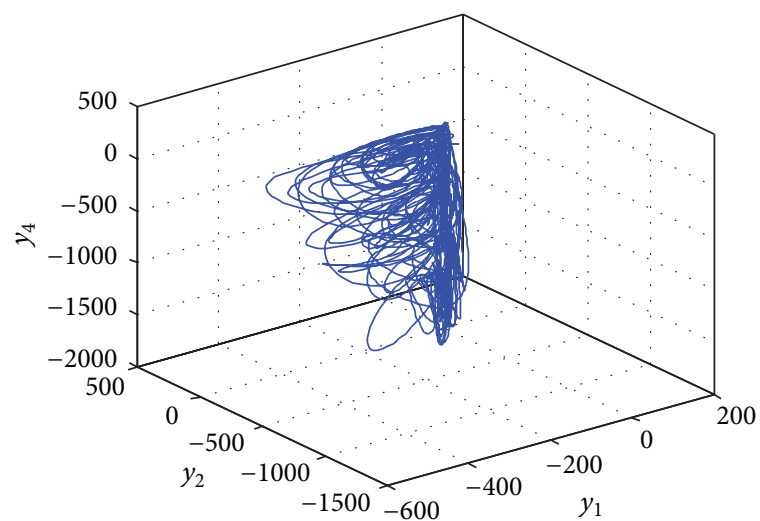

(b)

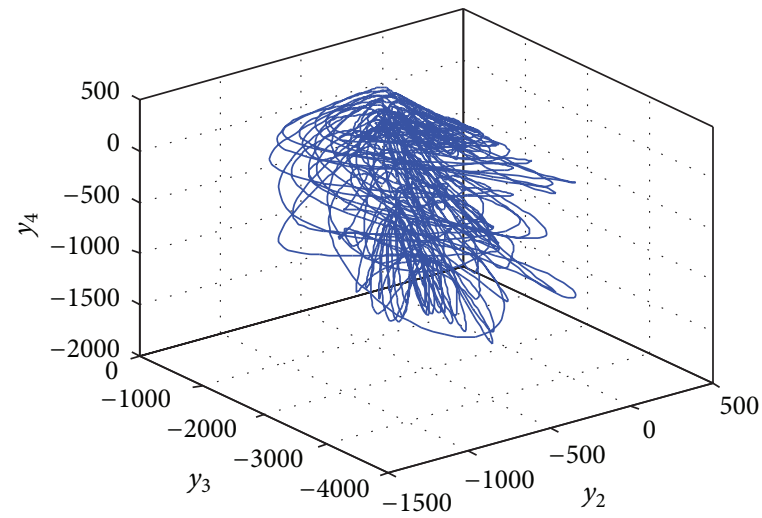

(d)

Figure 2: Projections of the phase portrait for chaotic system (3) of Case 1.

Its time derivative along any solution of (28) is

$$
\begin{aligned}
& \dot{V}=e_{1}\{[\left.2 x_{1}\left(A(t)\left(x_{2}-x_{1}\right)+R(t) x_{4}\right)+\left(\widehat{a}\left(y_{2}-y_{1}\right)+\widehat{r} y_{4}\right)\right] \\
& \times(\sin \omega t+3)+\omega \cos \omega t\left(x_{1}^{2}+y_{1}\right)-\widehat{a}\left(y_{2}-y_{1}\right) \\
&- \widehat{r} y_{4}-u_{1}+A(t)\left(x_{2}(t-T)-x_{1}(t-T)\right) \\
&+\left.R(t) x_{4}(t-T)\right\} \\
&+e_{2}\left\{\left[2 x_{2}\left(D(t) x_{1}-x_{2}-x_{1} x_{3}\right)+\left(\widehat{d} y_{1}-y_{2}-y_{1} y_{3}\right)\right]\right. \\
& \times(\sin \omega t+3)+\omega \cos \omega t\left(x_{2}^{2}+y_{2}\right)-\widehat{d} y_{1} \\
&+y_{2}+y_{1} y_{3}-u_{2}+D(t) x_{1}(t-T) \\
&\left.-x_{2}(t-T)-x_{1}(t-T) x_{3}(t-T)\right\} \\
&+e_{3}\left\{\left[2 x_{3}\left(-B(t) x_{3}+x_{1} x_{2}\right)-\widehat{b} y_{3}+y_{1} y_{2}\right]\right. \\
& \times(\sin \omega t+3)+\omega \cos \omega t\left(x_{3}^{2}+y_{3}\right)+\widehat{b} y_{3}-y_{1} y_{2} \\
&\left.-u_{3}-B(t) x_{3}(t-T)+x_{1}(t-T) x_{2}(t-T)\right\} \\
&+e_{4}\left\{\left[2 x_{4}\left(-x_{4}-C(t) x_{1}\right)-y_{4}-\widehat{c} y_{1}\right](\sin \omega t+3)\right. \\
&+\omega \cos \omega t\left(x_{4}^{2}+y_{4}\right)+y_{4}+\widehat{c} y_{1}-u_{4}-x_{4}(t-T) \\
&\left.-C(t) x_{1}(t-T)\right\}+\tilde{a} \tilde{a}+\tilde{b} \dot{\tilde{b}}+\tilde{c} \dot{c}+\tilde{d} \tilde{d}+\tilde{r} \dot{r} . \\
&
\end{aligned}
$$

The adaptive controllers are chosen as

$$
\begin{aligned}
u_{1}= & {\left[2 x_{1}\left(A(t)\left(x_{2}-x_{1}\right)+R(t) x_{4}\right)+\left(a\left(y_{2}-y_{1}\right)+r y_{4}\right)\right] } \\
& \times(\sin \omega t+3)+\omega \cos \omega t\left(x_{1}^{2}+y_{1}\right)-a\left(y_{2}-y_{1}\right) \\
& -r y_{4}+A(t)\left(x_{2}(t-T)-x_{1}(t-T)\right) \\
& +R(t) x_{4}(t-T)+e_{1}, \\
u_{2}= & {\left[2 x_{2}\left(D(t) x_{1}-x_{2}-x_{1} x_{3}\right)+\left(d y_{1}-y_{2}-y_{1} y_{3}\right)\right] } \\
& \times(\sin \omega t+3)+\omega \cos \omega t\left(x_{2}^{2}+y_{2}\right)-d y_{1} \\
& +y_{2}+y_{1} y_{3}+D(t) x_{1}(t-T)-x_{2}(t-T) \\
& -x_{1}(t-T) x_{3}(t-T)+e_{2}, \\
u_{3}= & {\left[2 x_{3}\left(-B(t) x_{3}+x_{1} x_{2}\right)-b y_{3}+y_{1} y_{2}\right](\sin \omega t+3) } \\
& +\omega \cos \omega t\left(x_{3}^{2}+y_{3}\right)+b y_{3}-y_{1} y_{2} \\
& -B(t) x_{3}(t-T)+x_{1}(t-T) x_{2}(t-T)+e_{3}, \\
u_{4}= & {\left[2 x_{4}\left(-x_{4}-C(t) x_{1}\right)-y_{4}-c y_{1}\right](\sin \omega t+3) } \\
& +\omega \cos \omega t\left(x_{4}^{2}+y_{4}\right)+y_{4}+c y_{1}-x_{4}(t-T) \\
& -C(t) x_{1}(t-T)+e_{4},
\end{aligned}
$$




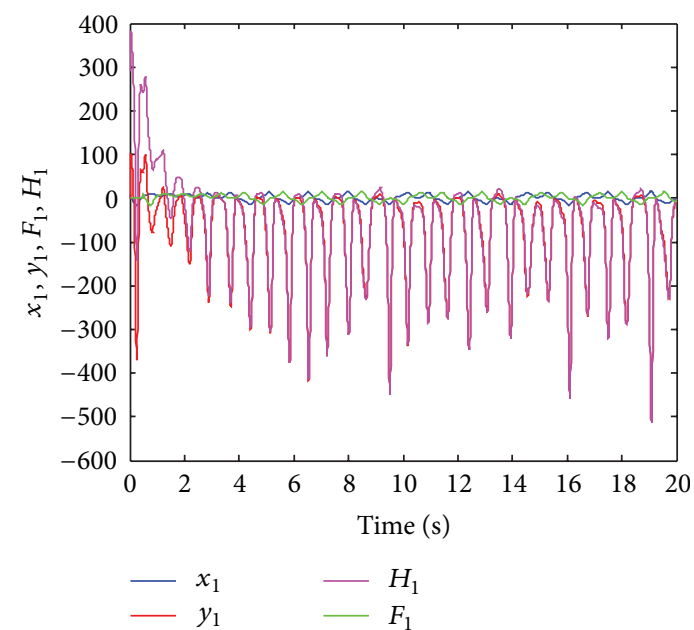

(a)

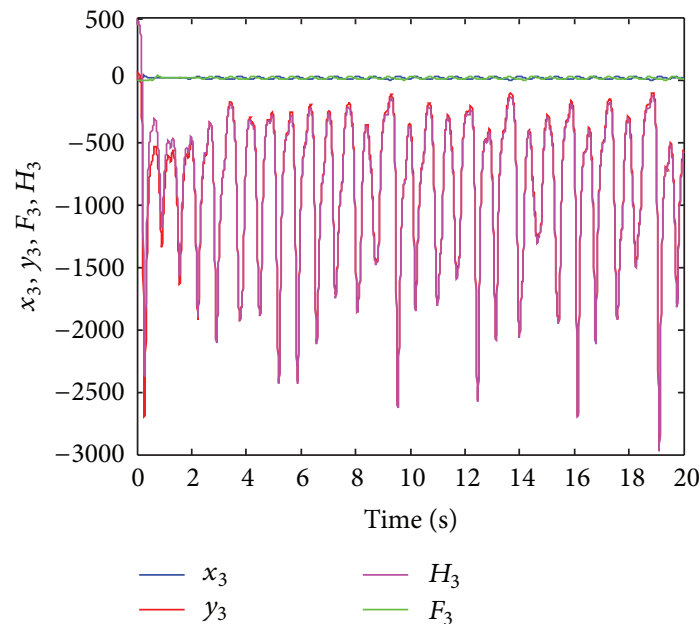

(c)

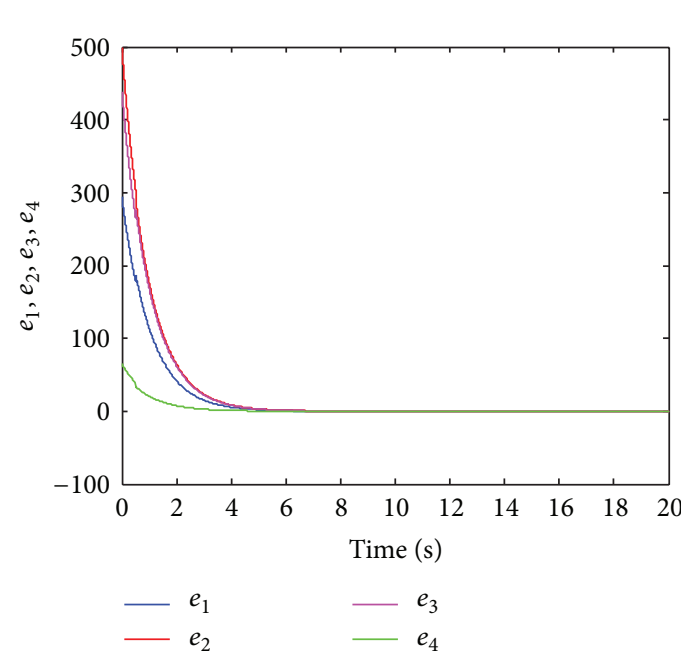

(e)

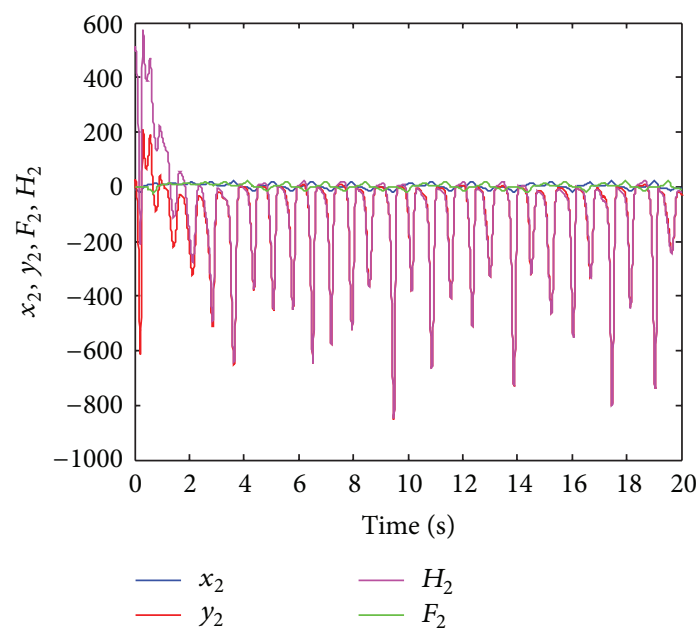

(b)
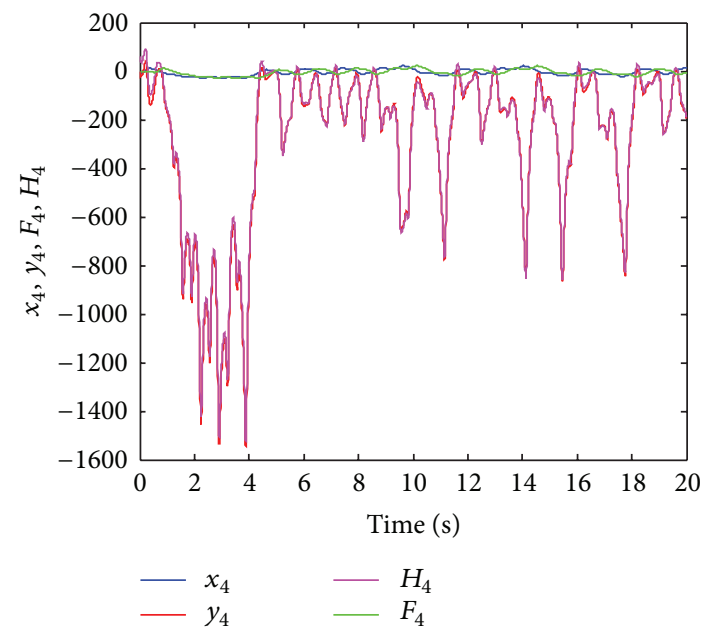

(d)

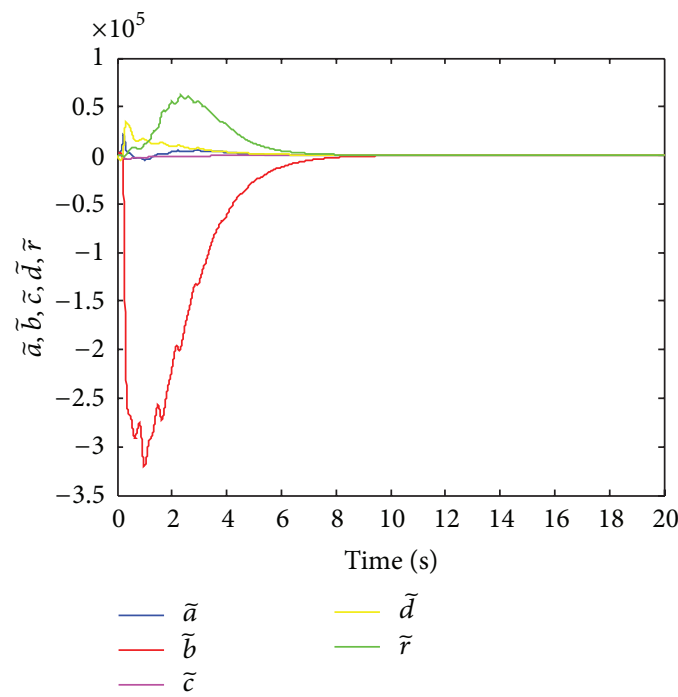

(f)

FigURE 3: Time histories of states, state errors, parameter differences, $F_{1}, F_{2}, F_{3}, F_{4}, H_{1}, H_{2}, H_{3}$, and $H_{4}$ for Case 1. 


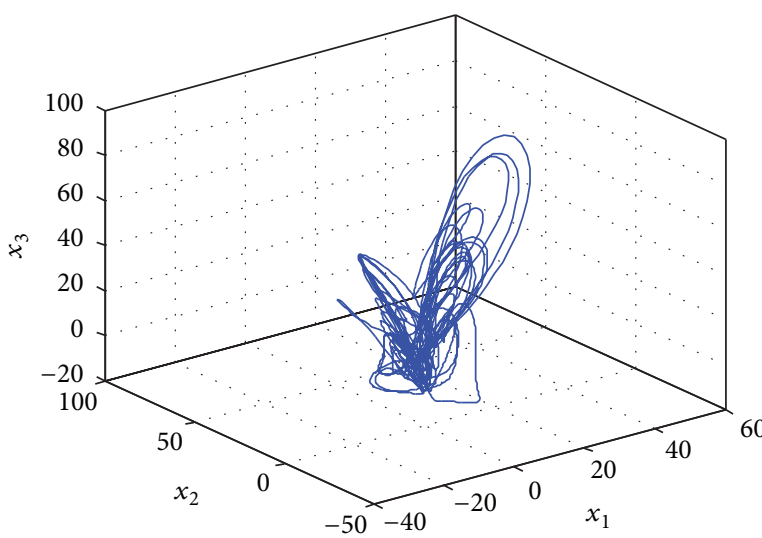

(a)

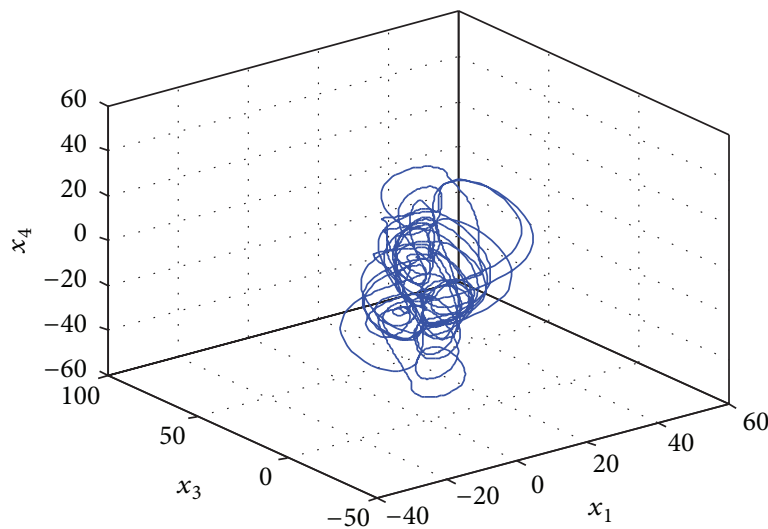

(c)

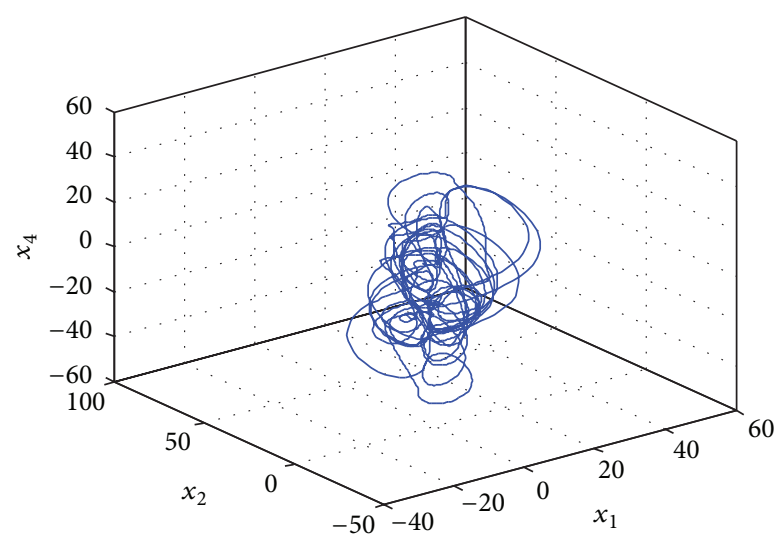

(b)

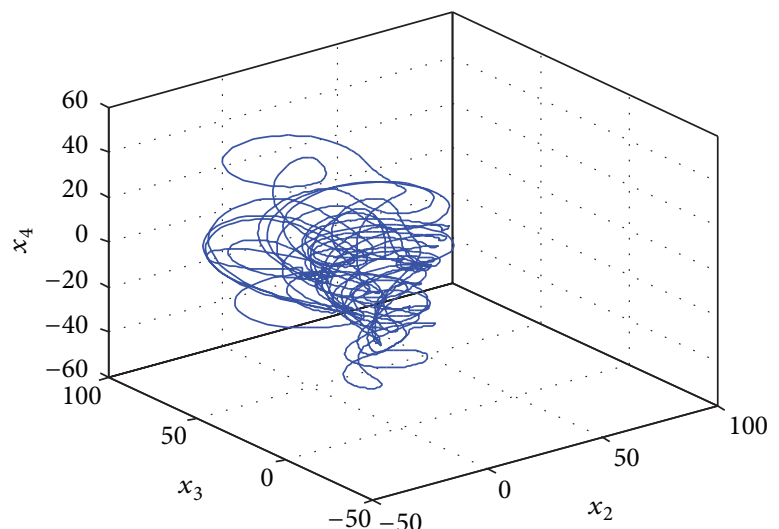

(d)

Figure 4: Projections of the phase portrait for chaotic system (23).

and the update laws are chosen as

$$
\begin{aligned}
\dot{\tilde{a}}=2 x_{1} & \left(y_{2}-y_{1}\right)(\sin \omega t+3) e_{1}+\left(y_{2}-y_{1}\right) e_{1}-\tilde{a}, \\
\dot{\tilde{b}} & =-2 x_{3} y_{3} e_{3}(\sin \omega t+3)-y_{3} e_{3}-\tilde{b}, \\
\dot{\tilde{c}} & =-2 x_{4} y_{1} e_{4}(\sin \omega t+3)-y_{1} e_{4}-\tilde{c}, \\
\dot{\tilde{d}} & =2 x_{2} y_{1} e_{2}(\sin \omega t+3)+y_{1} e_{2}-\tilde{d}, \\
& \dot{\tilde{r}}=2 x_{1} y_{4} e_{1}(\sin \omega t+3)+y_{4} e_{1}-\tilde{r} .
\end{aligned}
$$

The initial values of estimate for uncertain parameters are $\widehat{a}(0)=\widehat{b}(0)=\widehat{c}(0)=\widehat{d}(0)=\widehat{r}(0)=0$.

Equation (31) becomes

$$
\dot{V}=-\left(e_{1}^{2}+e_{2}^{2}+e_{3}^{2}+e_{4}^{2}+\widetilde{a}^{2}+\widetilde{b}^{2}+\widetilde{c}^{2}+\widetilde{d}^{2}+\widetilde{r}^{2}\right)<0,
$$

which is negative definite. The Lyapunov asymptotical stability theorem is satisfied. The time delay symplectic synchronization with uncertain chaotic parameters of the identical Lorenz-Stenflo systems is achieved. The numerical results of the phase portrait of chaotic system (3), the time series of states, state errors, parameters, and parameter differences are shown in Figures 5, 6, and 7, respectively. The symplectic chaos synchronization is accomplished using adaptive control method.

Case 3. A multitime delay symplectic synchronization with uncertain chaotic parameters.

We take $F_{1}(t)=x_{1}\left(t-T_{1}\right), F_{2}(t)=x_{2}\left(t-T_{2}\right), F_{3}(t)=$ $x_{3}\left(t-T_{3}\right)$, and $F_{4}(t)=x_{4}\left(t-T_{4}\right)$. They are chaotic functions of time, where multi time delay $T_{1}, T_{2}, T_{3}$, and $T_{4}$ are positive constants, $T_{1}=0.5 \mathrm{sec}, T_{2}=0.7 \mathrm{sec}, T_{3}=0.8 \mathrm{sec}$, and $T_{4}=$ 0.9 sec. The $H_{i}(x, y, t)=\left(x_{i}^{2}+y_{i}\right)(\sin w t+3)(i=1,2,3,4)$ are given. By (5) we have

$$
\begin{array}{r}
\lim _{t \rightarrow \infty} e_{i}=\lim _{t \rightarrow \infty}\left(\left(x_{i}^{2}+y_{i}\right)(\sin \omega t+3)-y_{i}+x_{i}\left(t-T_{i}\right)\right)=0, \\
i=1,2,3,4 .
\end{array}
$$

From (35) we have

$$
\begin{aligned}
\dot{e}_{i}= & \left(2 x_{i} \dot{x}_{i}+\dot{y}_{i}\right)(\sin \omega t+3)+\omega \cos \omega t\left(x_{i}^{2}+y_{i}\right) \\
& -\dot{y}_{i}+\dot{x}_{i}\left(t-T_{i}\right), \quad i=1,2,3,4 .
\end{aligned}
$$




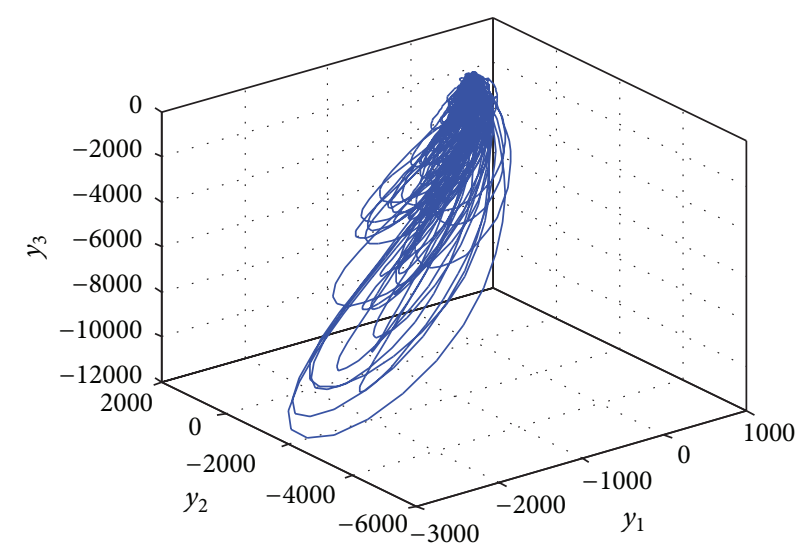

(a)

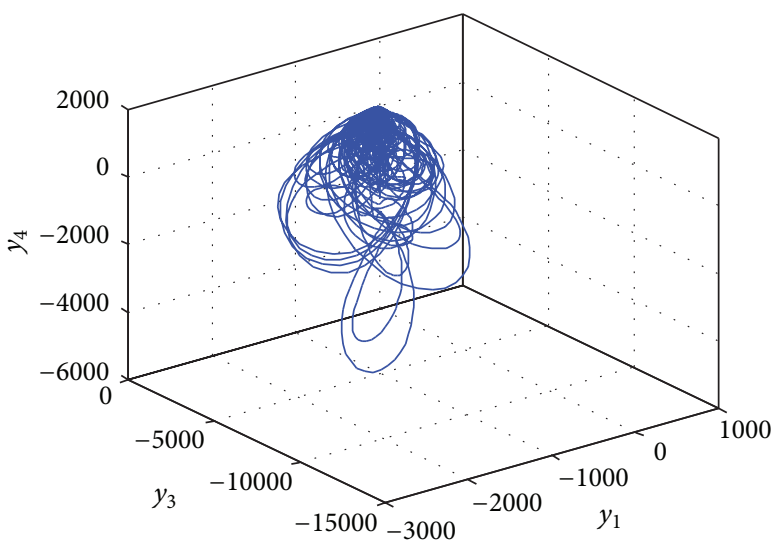

(c)

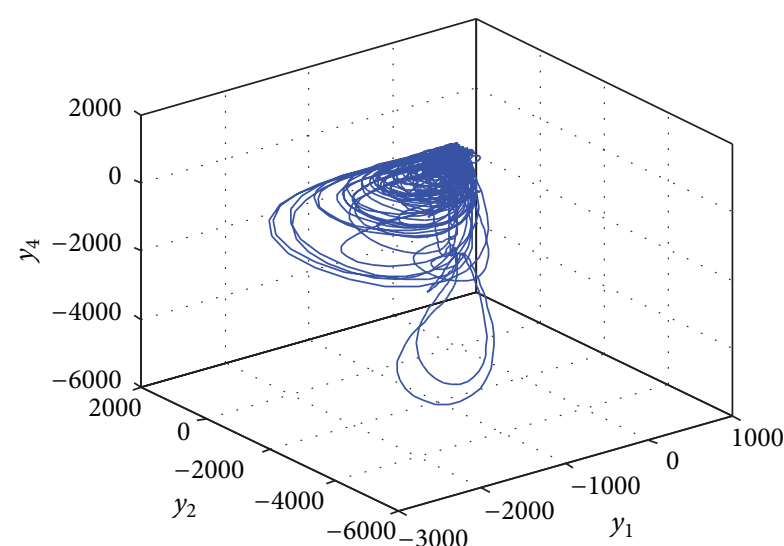

(b)

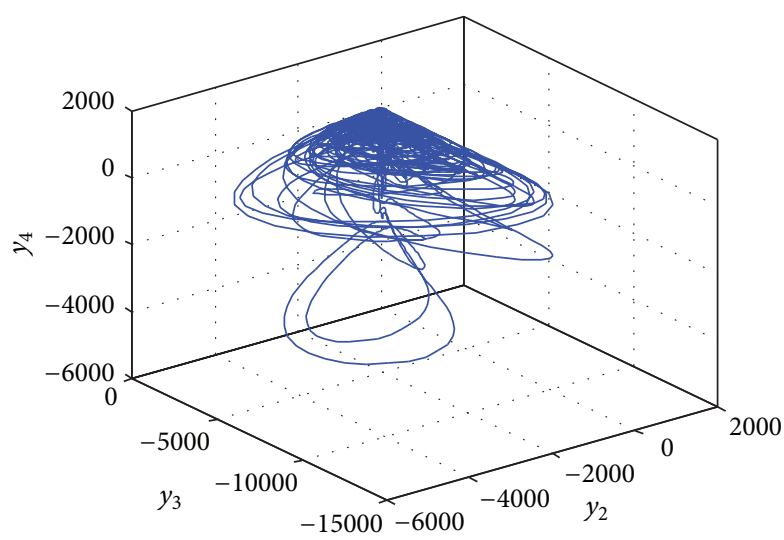

(d)

FIgURE 5: Projections of the phase portrait for chaotic system (3) of Case 2.

Equation (36) can be expressed as

$$
\begin{aligned}
\dot{e}_{1}= & {\left[2 x_{1}\left(A(t)\left(x_{2}-x_{1}\right)+R(t) x_{4}\right)+\left(\widehat{a}\left(y_{2}-y_{1}\right)+\widehat{r} y_{4}\right)\right] } \\
& \times(\sin \omega t+3)+\omega \cos \omega t\left(x_{1}^{2}+y_{1}\right)-\widehat{a}\left(y_{2}-y_{1}\right) \\
& -\widehat{r} y_{4}-u_{1}+A(t)\left(x_{2}\left(t-T_{2}\right)-x_{1}\left(t-T_{1}\right)\right) \\
& +R(t) x_{4}\left(t-T_{4}\right), \\
\dot{e}_{2}= & {\left[2 x_{2}\left(D(t) x_{1}-x_{2}-x_{1} x_{3}\right)+\left(\widehat{d} y_{1}-y_{2}-y_{1} y_{3}\right)\right] } \\
& \times(\sin \omega t+3)+\omega \cos \omega t\left(x_{2}^{2}+y_{2}\right)-\widehat{d} y_{1} \\
& +y_{2}+y_{1} y_{3}+u_{2}+D(t) x_{1}\left(t-T_{1}\right) \\
& -x_{2}\left(t-T_{2}\right)-x_{1}\left(t-T_{1}\right) x_{3}\left(t-T_{3}\right), \\
\dot{e}_{3}= & {\left[2 x_{3}\left(-B(t) x_{3}+x_{1} x_{2}\right)-\widehat{b} y_{3}+y_{1} y_{2}\right] } \\
& \times(\sin \omega t+3)+\omega \cos \omega t\left(x_{3}^{2}+y_{3}\right) \\
& +\widehat{b} y_{3}-y_{1} y_{2}-u_{3}-B(t) x_{3}\left(t-T_{3}\right) \\
& +x_{1}\left(t-T_{1}\right) x_{2}\left(t-T_{2}\right),
\end{aligned}
$$

$$
\begin{aligned}
\dot{e}_{4}= & {\left[2 x_{4}\left(-x_{4}-C(t) x_{1}\right)-y_{4}-\widehat{c} y_{1}\right](\sin \omega t+3) } \\
& +\omega \cos \omega t\left(x_{4}^{2}+y_{4}\right)+y_{4}+\widehat{c} y_{1}-u_{4} \\
& -x_{4}\left(t-T_{4}\right)-C(t) x_{1}\left(t-T_{1}\right)
\end{aligned}
$$

where

$$
\begin{aligned}
& e_{1}=\left(x_{1}^{2}+y_{1}\right)(\sin \omega t+3)-y_{1}+x_{1}\left(t-T_{1}\right), \\
& e_{2}=\left(x_{2}^{2}+y_{2}\right)(\sin \omega t+3)-y_{2}+x_{2}\left(t-T_{2}\right), \\
& e_{3}=\left(x_{3}^{2}+y_{3}\right)(\sin \omega t+3)-y_{3}+x_{3}\left(t-T_{3}\right), \\
& e_{4}=\left(x_{4}^{2}+y_{4}\right)(\sin \omega t+3)-y_{4}+x_{4}\left(t-T_{4}\right) .
\end{aligned}
$$

Choose a positive definite Lyapunov function:

$$
\begin{aligned}
& V\left(e_{1}, e_{2}, e_{3}, e_{4}, \tilde{a}, \tilde{b}, \widetilde{c}, \tilde{d}, \widetilde{r}\right) \\
& \quad=\frac{1}{2}\left(e_{1}^{2}+e_{2}^{2}+e_{3}^{2}+e_{4}^{2}+\widetilde{a}^{2}+\widetilde{b}^{2}+\widetilde{c}^{2}+\widetilde{d}^{2}+\widetilde{r}^{2}\right),
\end{aligned}
$$




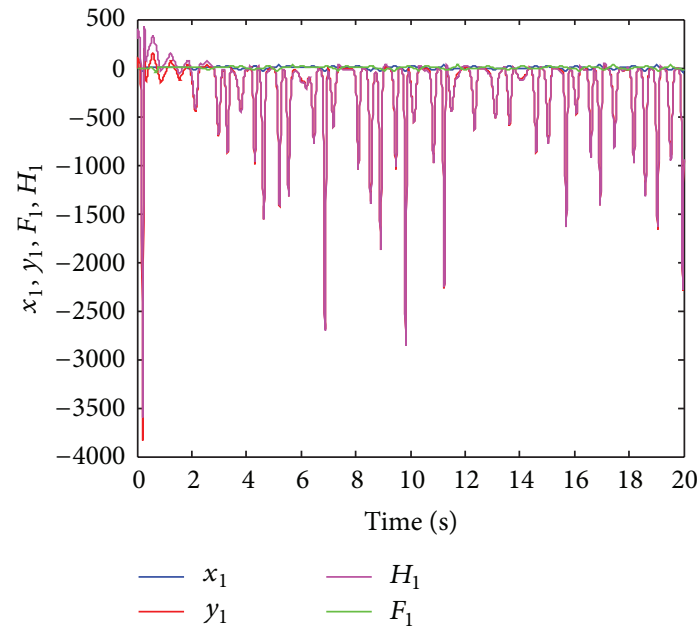

(a)

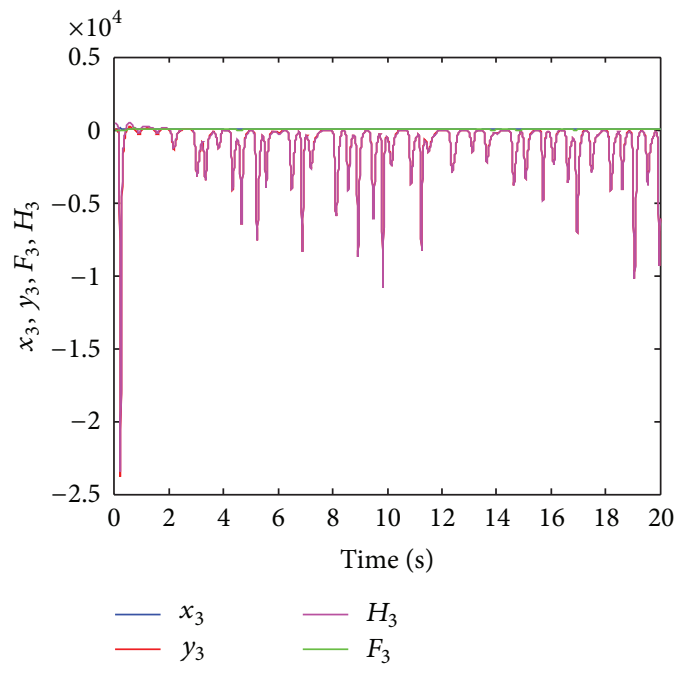

(c)

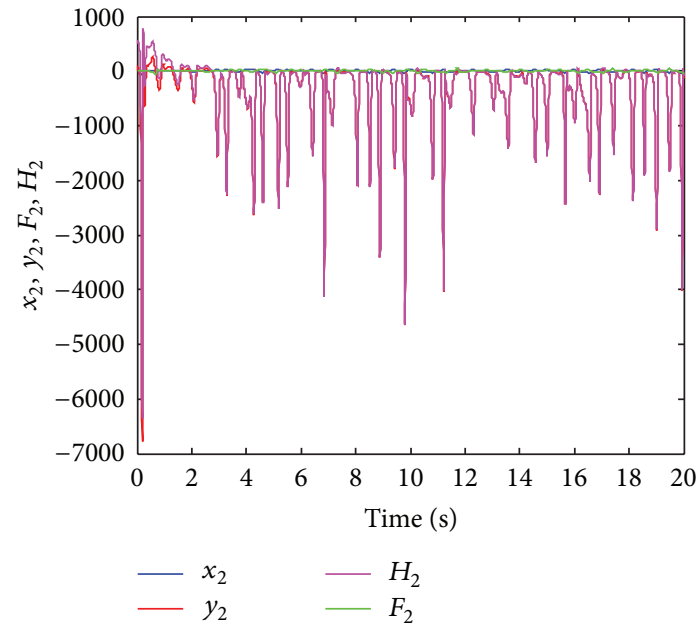

(b)
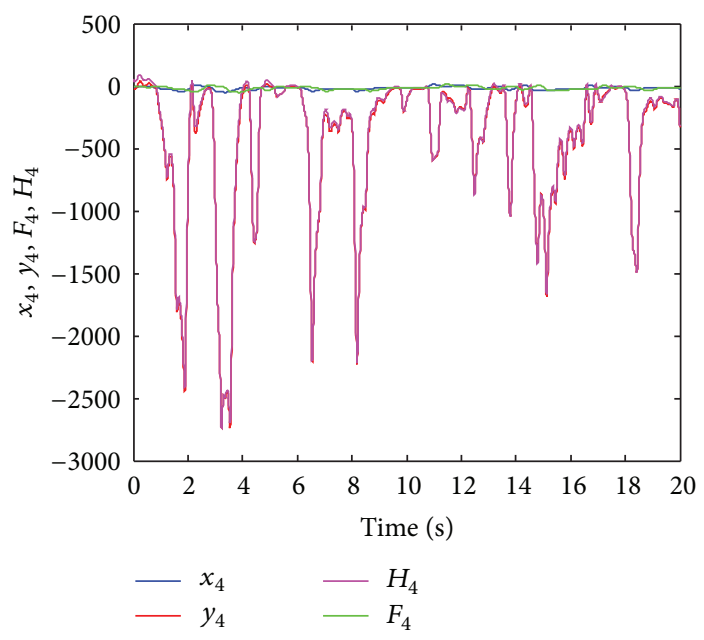

(d)

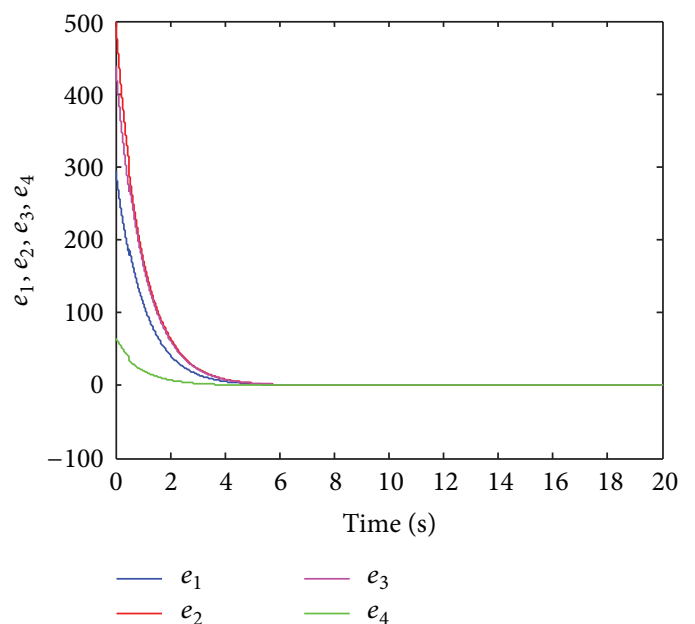

(e)

FIgURE 6: Time histories of states, state errors, $F_{1}, F_{2}, F_{3}, F_{4}, H_{1}, H_{2}, H_{3}$, and $H_{4}$ for Case 2. 


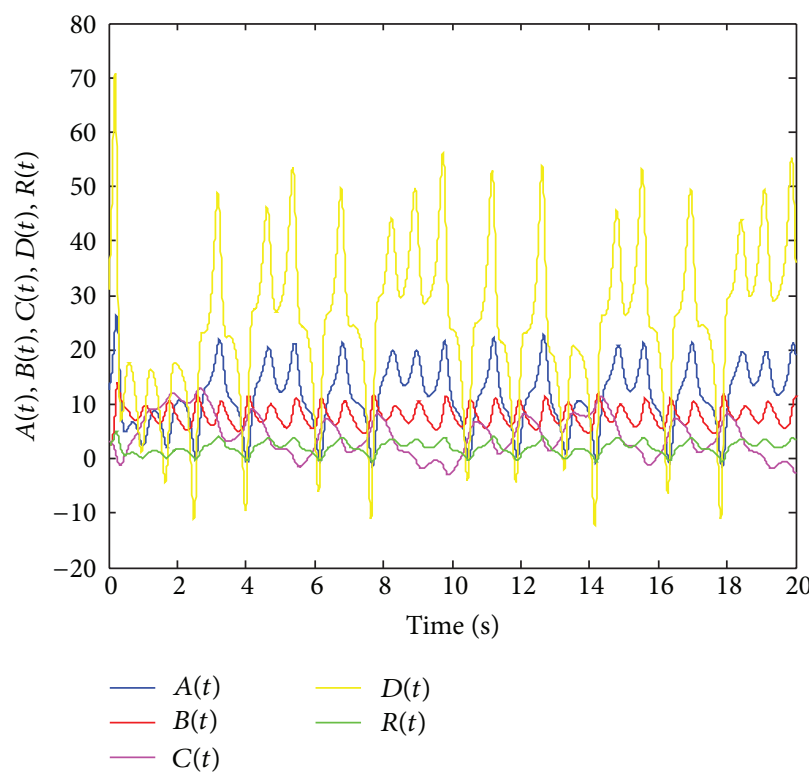

(a)

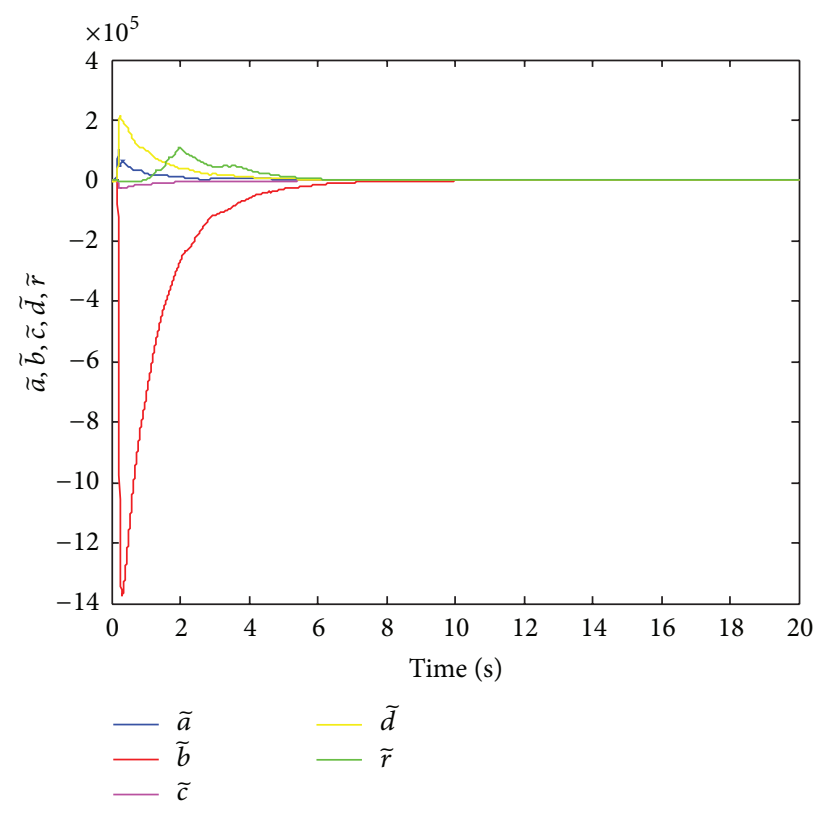

(b)

Figure 7: Time histories of $A(t), B(t), C(t), D(t)$, and $R(t)$ and parameter differences for Case 2 .

where $\widetilde{a}=A(t)-\widehat{a}, \widetilde{b}=B(t)-\widehat{b}, \widetilde{c}=C(t)-\widehat{c}, \widetilde{d}=D(t)-\widehat{d}$, $\widetilde{r}=R(t)-\widehat{r}$, and $\widehat{a}, \widehat{b}, \widehat{c}, \widehat{d}$, and $\widehat{r}$ are estimates of uncertain parameters $A(t), B(t), C(t), D(t)$, and $R(t)$, respectively.

Its time derivative along any solution of (37) is

$$
\begin{aligned}
\dot{V}=e_{1}\{ & {\left[2 x_{1}\left(A(t)\left(x_{2}-x_{1}\right)+R(t) x_{4}\right)+\left(\hat{a}\left(y_{2}-y_{1}\right)+\widehat{r} y_{4}\right)\right] } \\
\times & (\sin \omega t+3)+\omega \cos \omega t\left(x_{1}^{2}+y_{1}\right)-\widehat{a}\left(y_{2}-y_{1}\right) \\
- & \widehat{r} y_{4}-u_{1}+A(t)\left(x_{2}\left(t-T_{2}\right)-x_{1}\left(t-T_{1}\right)\right) \\
+ & \left.R(t) x_{4}\left(t-T_{4}\right)\right\} \\
+e_{2} & \left\{\left[2 x_{2}\left(D(t) x_{1}-x_{2}-x_{1} x_{3}\right)+\left(\widehat{d} y_{1}-y_{2}-y_{1} y_{3}\right)\right]\right. \\
& \times(\sin \omega t+3)+\omega \cos \omega t\left(x_{2}^{2}+y_{2}\right)-\widehat{d} y_{1}+y_{2} \\
& +y_{1} y_{3}-u_{2}+D(t) x_{1}\left(t-T_{1}\right)-x_{2}\left(t-T_{2}\right) \\
& \left.-x_{1}\left(t-T_{1}\right) x_{3}\left(t-T_{3}\right)\right\} \\
+e_{3} & \left\{\left[2 x_{3}\left(-B(t) x_{3}+x_{1} x_{2}\right)-\widehat{b} y_{3}+y_{1} y_{2}\right](\sin \omega t+3)\right. \\
& +\omega \cos \omega t\left(x_{3}^{2}+y_{3}\right)+\widehat{b} y_{3}-y_{1} y_{2}-u_{3} \\
& \left.-B(t) x_{3}\left(t-T_{3}\right)+x_{1}\left(t-T_{1}\right) x_{2}\left(t-T_{2}\right)\right\} \\
+e_{4} & \left\{\left[2 x_{4}\left(-x_{4}-C(t) x_{1}\right)-y_{4}-\widehat{c} y_{1}\right](\sin \omega t+3)\right. \\
& +\omega \cos \omega t\left(x_{4}^{2}+y_{4}\right)+y_{4}+\widehat{c} y_{1}-u_{4}-x_{4}\left(t-T_{4}\right) \\
& \left.-C(t) x_{1}\left(t-T_{1}\right)\right\}+\tilde{a} \dot{\tilde{a}}+\tilde{b} \dot{\vec{b}}+\dot{\vec{c} \dot{c}}+\tilde{d} \dot{d}+\tilde{r} \dot{r} .
\end{aligned}
$$

The adaptive controllers are chosen as

$$
\begin{aligned}
u_{1}= & {\left[2 x_{1}\left(A(t)\left(x_{2}-x_{1}\right)+R(t) x_{4}\right)+\left(a\left(y_{2}-y_{1}\right)+r y_{4}\right)\right] } \\
& \times(\sin \omega t+3)+\omega \cos \omega t\left(x_{1}^{2}+y_{1}\right)-a\left(y_{2}-y_{1}\right) \\
& -r y_{4}+A(t)\left(x_{2}\left(t-T_{2}\right)-x_{1}\left(t-T_{1}\right)\right) \\
& +R(t) x_{4}\left(t-T_{4}\right)+e_{1}, \\
u_{2}= & {\left[2 x_{2}\left(D(t) x_{1}-x_{2}-x_{1} x_{3}\right)+\left(d y_{1}-y_{2}-y_{1} y_{3}\right)\right] } \\
& \times(\sin \omega t+3)+\omega \cos \omega t\left(x_{2}^{2}+y_{2}\right)-d y_{1}+y_{2} \\
& +y_{1} y_{3}+D(t) x_{1}\left(t-T_{1}\right)-x_{2}\left(t-T_{2}\right) \\
& -x_{1}\left(t-T_{1}\right) x_{3}\left(t-T_{3}\right)+e_{2}, \\
u_{3}= & {\left[2 x_{3}\left(-B(t) x_{3}+x_{1} x_{2}\right)-b y_{3}+y_{1} y_{2}\right](\sin \omega t+3) } \\
& +\omega \cos \omega t\left(x_{3}^{2}+y_{3}\right)+b y_{3}-y_{1} y_{2} \\
& -B(t) x_{3}\left(t-T_{3}\right)+x_{1}\left(t-T_{1}\right) x_{2}\left(t-T_{2}\right)+e_{3}, \\
u_{4}= & {\left[2 x_{4}\left(-x_{4}-C(t) x_{1}\right)-y_{4}-c y_{1}\right](\sin \omega t+3) } \\
& +\omega \cos \omega t\left(x_{4}^{2}+y_{4}\right)+y_{4}+c y_{1} \\
& -x_{4}\left(t-T_{4}\right)-C(t) x_{1}\left(t-T_{1}\right)+e_{4},
\end{aligned}
$$




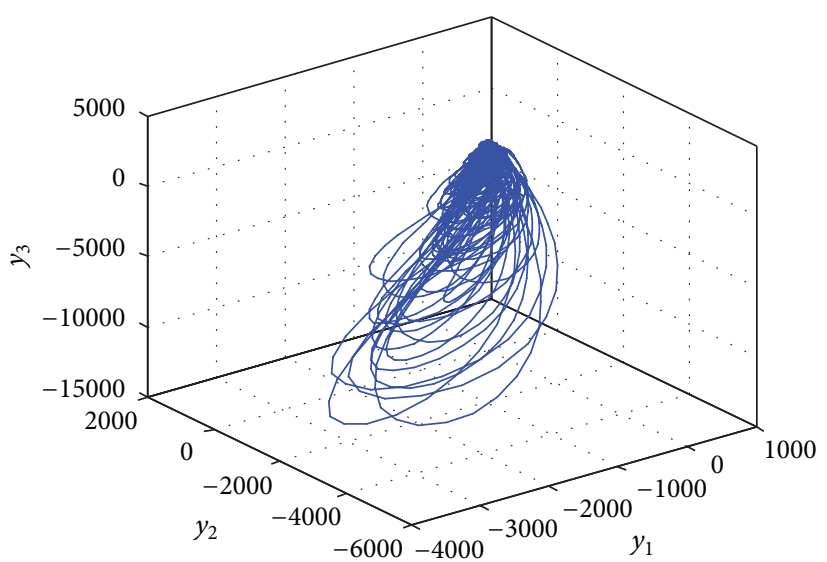

(a)

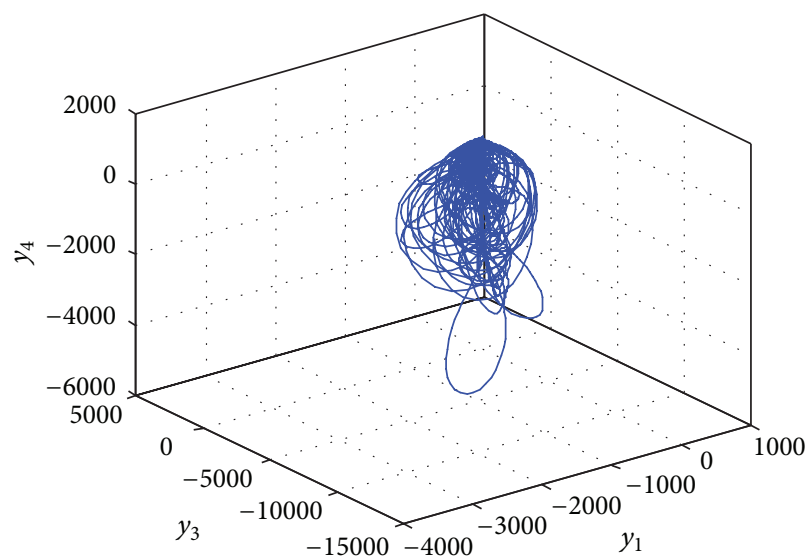

(c)

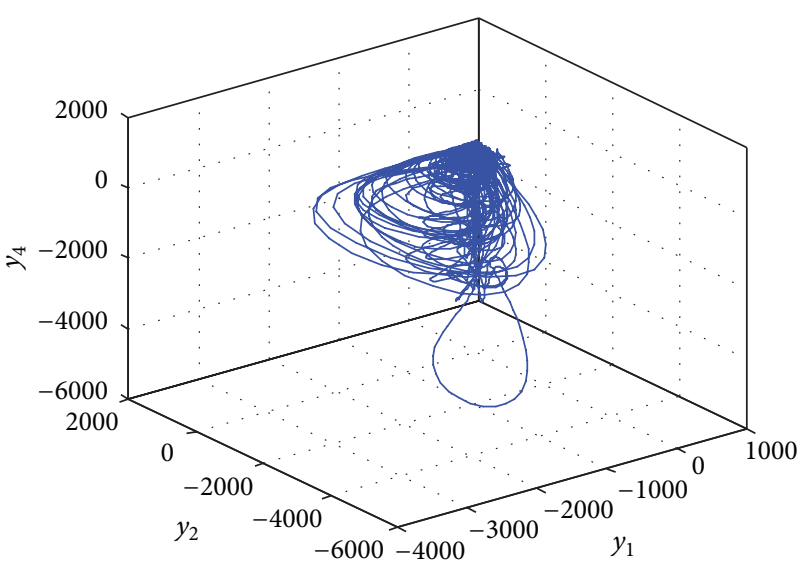

(b)

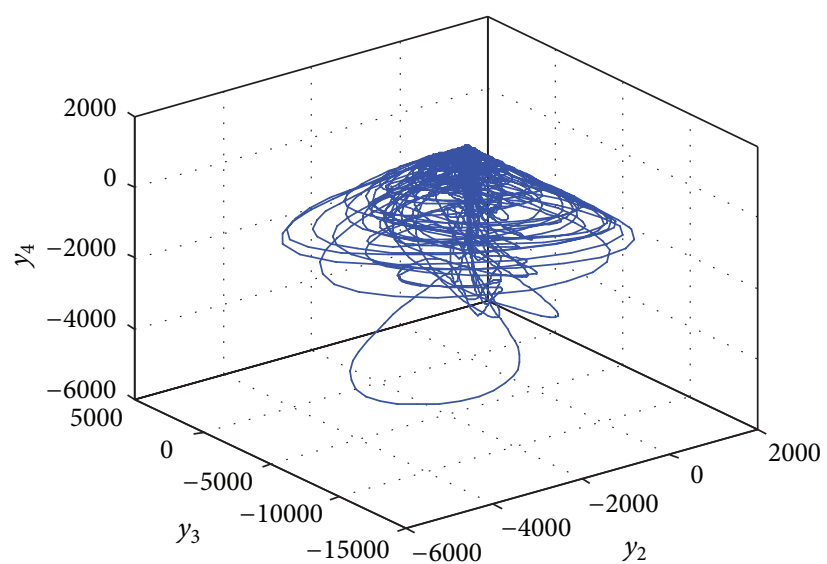

(d)

FIGURE 8: Projections of the phase portrait for chaotic system (3) of Case 3.

and the update laws are chosen as

$$
\begin{gathered}
\dot{\tilde{a}}=2 x_{1}\left(y_{2}-y_{1}\right)(\sin \omega t+3) e_{1}+\left(y_{2}-y_{1}\right) e_{1}-\tilde{a}, \\
\dot{\widetilde{b}}=-2 x_{3} y_{3} e_{3}(\sin \omega t+3)-y_{3} e_{3}-\tilde{b}, \\
\dot{\tilde{c}}=-2 x_{4} y_{1} e_{4}(\sin \omega t+3)-y_{1} e_{4}-\tilde{c}, \\
\dot{\widetilde{d}}=2 x_{2} y_{1} e_{2}(\sin \omega t+3)+y_{1} e_{2}-\tilde{d}, \\
\dot{\tilde{r}}=2 x_{1} y_{4} e_{1}(\sin \omega t+3)+y_{4} e_{1}-\tilde{r} .
\end{gathered}
$$

The initial values of estimate for uncertain parameters are $\widehat{a}(0)=\widehat{b}(0)=\widehat{c}(0)=\widehat{d}(0)=\widehat{r}(0)=0$.

Equation (40) becomes

$$
\dot{V}=-\left(e_{1}^{2}+e_{2}^{2}+e_{3}^{2}+e_{4}^{2}+\widetilde{a}^{2}+\widetilde{b}^{2}+\widetilde{c}^{2}+\widetilde{d}^{2}+\widetilde{r}^{2}\right)<0,
$$

which is negative definite. The Lyapunov asymptotical stability theorem is satisfied. The multi time delay symplectic synchronization with uncertain chaotic parameters of the identical Lorenz-Stenflo systems is achieved. The numerical results of the phase portrait of chaotic system (3), the time series of states, state errors, and parameter differences are shown in Figures 8 and 9, respectively. The symplectic chaos synchronization is accomplished using adaptive control method.

\section{Conclusions}

A novel symplectic synchronization of a Lorenz-Stenflo system with uncertain chaotic parameters is obtained by the Lyapunov asymptotical stability theorem. The simulation results of three cases are shown in corresponding figures which imply that the adaptive controllers and update laws we designed are feasible and effective. The symplectic synchronization of chaotic systems with uncertain chaotic parameters via adaptive control concept can be used to increase the security of secret communication system. 

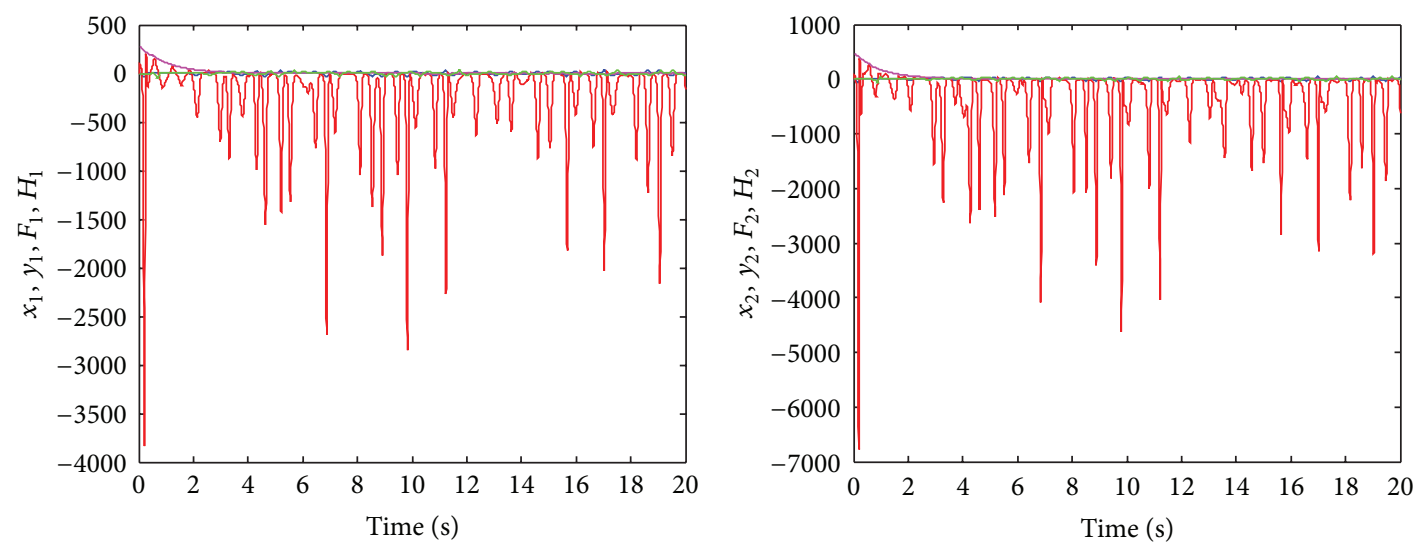

$\begin{array}{ll}x_{1} & -H_{1} \\ y_{1} & -F_{1}\end{array}$

(a)
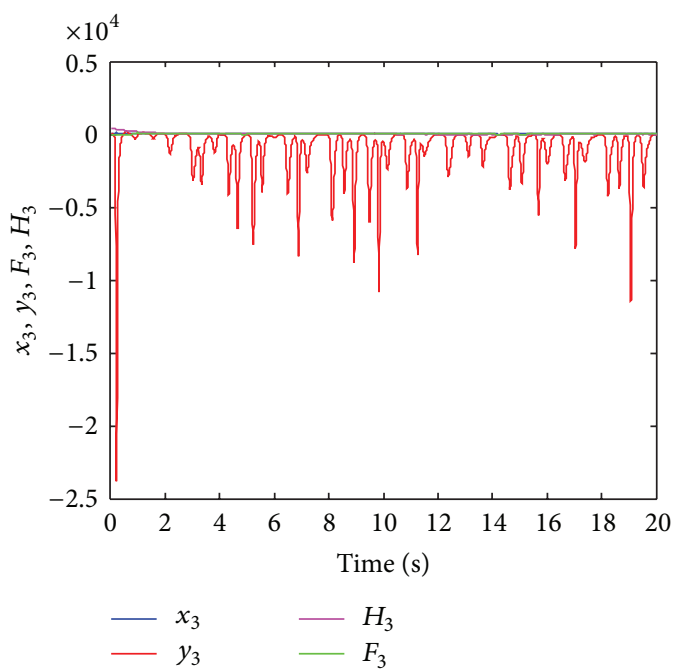

(c)

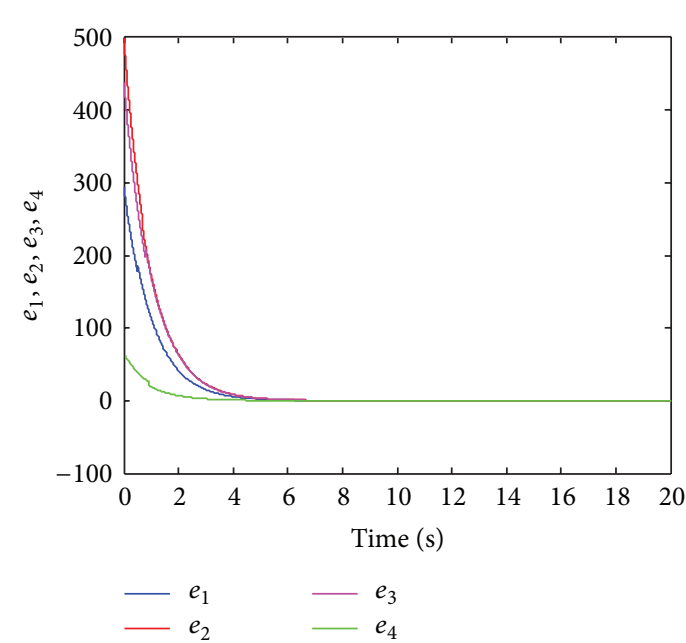

(e)

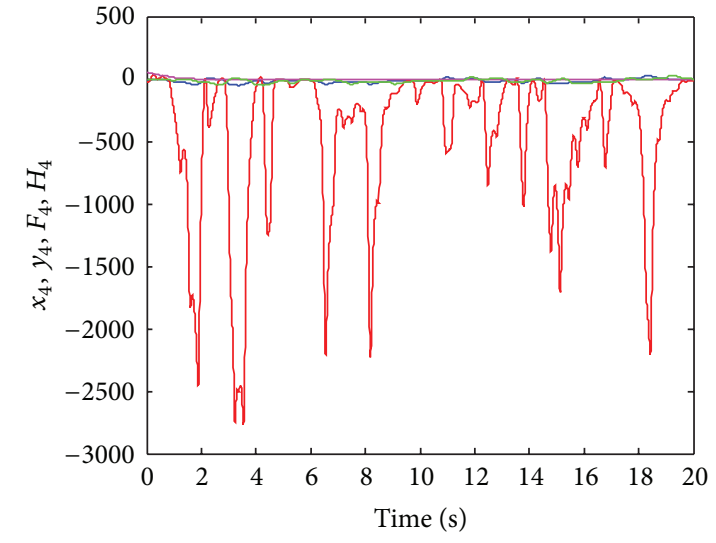

$-x_{4} \quad-H_{4}$

(d)

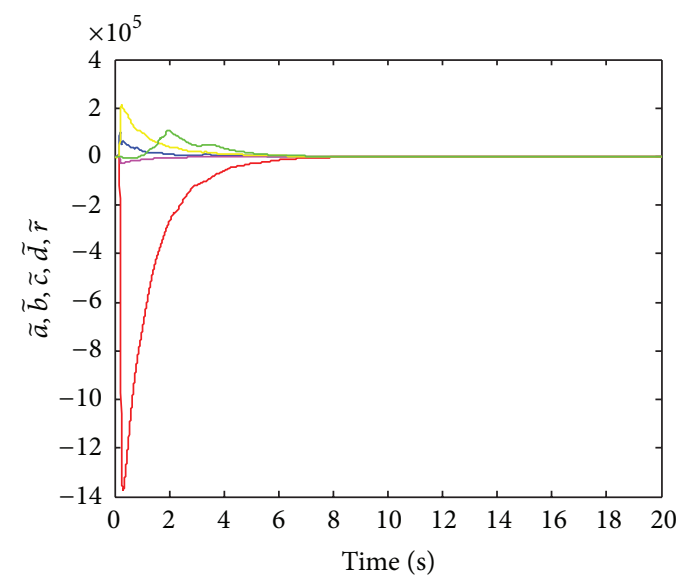

(f)

FiguRE 9: Time histories of states, state errors, parameter differences, $F_{1}, F_{2}, F_{3}, F_{4}, H_{1}, H_{2}, H_{3}$, and $H_{4}$ for Case 3. 


\section{Acknowledgment}

This research was supported by the National Science Council, Republic of China, under Grant no. 98-2218-E-011-010.

\section{References}

[1] R. Femat and G. Solís-Perales, "On the chaos synchronization phenomena," Physics Letters A, vol. 262, no. 1, pp. 50-60, 1999.

[2] Z.-M. Ge and C.-H. Yang, "Pragmatical generalized synchronization of chaotic systems with uncertain parameters by adaptive control," Physica D, vol. 231, no. 2, pp. 87-94, 2007.

[3] S. S. Yang and C. K. Duan, "Generalized synchronization in chaotic systems," Chaos, Solitons \& Fractals, vol. 9, no. 10, pp. 1703-1707, 1998.

[4] A. Krawiecki and A. Sukiennicki, "Generalizations of the concept of marginal synchronization of chaos," Chaos, Solitons \& Fractals, vol. 11, no. 9, pp. 1445-1458, 2000.

[5] Z. M. Ge, C. H. Yang, H. H. Chen, and S. C. Lee, "Nonlinear dynamics and chaos control of a physical pendulum with vibrating and rotating support," Journal of Sound and Vibration, vol. 242, no. 2, pp. 247-264, 2001.

[6] M. Y. Chen, Z. Z. Han, and Y. Shang, "General synchronization of Genesio-Tesi systems," International Journal of Bifurcation and Chaos, vol. 14, no. 1, pp. 347-354, 2004.

[7] Z. M. Ge and C. H. Yang, "Symplectic synchronization of different chaotic systems," Chaos, Solitons \& Fractals, vol. 40, no. 5, pp. 2532-2543, 2009.

[8] Z. M. Ge and C. H. Yang, "The generalized synchronization of a Quantum-CNN chaotic oscillator with different order systems," Chaos, Solitons \& Fractals, vol. 35, no. 5, pp. 980-990, 2008.

[9] Z.-M. Ge and C.-H. Yang, "Synchronization of complex chaotic systems in series expansion form," Chaos, Solitons \& Fractals, vol. 34, no. 5, pp. 1649-1658, 2007.

[10] L. M. Pecora and T. L. Carroll, "Synchronization in chaotic systems," Physical Review Letters, vol. 64, no. 8, pp. 821-824, 1990.

[11] L. Stenflo, "Generalized Lorenz equations for acoustic-gravity waves in the atmosphere," Physica Scripta, vol. 53, no. 1, pp. 8384, 1996.

[12] U. E. Vincent, "Synchronization of identical and non-identical 4-D chaotic systems using active control," Chaos, Solitons \& Fractals, vol. 37, no. 4, pp. 1065-1075, 2008.

[13] Z. Liu, "The first integrals of nonlinear acoustic gravity wave equations," Physica Scripta, vol. 61, no. 5, p. 526, 2000.

[14] C. Zhou, C. H. Lai, and M. Y. Yu, "Bifurcation behavior of the generalized Lorenz equations at large rotation numbers," Journal of Mathematical Physics, vol. 38, no. 10, pp. 5225-5239, 1997.

[15] S. Banerjee, P. Saha, and A. R. Chowdhury, "Chaotic scenario in the Stenflo equations," Physica Scripta, vol. 63, no. 3, pp. 177-180, 2001.

[16] Y. Chen, X. Wu, and Z. Gui, "Global synchronization criteria for two Lorenz-Stenflo systems via single-variable substitution control," Nonlinear Dynamics, vol. 62, no. 1-2, pp. 361-369, 2010.

[17] Z.-M. Ge and Y.-S. Chen, "Synchronization of unidirectional coupled chaotic systems via partial stability," Chaos, Solitons \& Fractals, vol. 21, no. 1, pp. 101-111, 2004.

[18] C. H. Yang, S. Y. Li, and P. C. Tsen, "Synchronization of chaotic system with uncertain variable parameters by linear coupling and pragmatical adaptive tracking," Nonlinear Dynamics, vol. 70, no. 3, pp. 2187-2202, 2012.

[19] Z. M. Ge and C. C. Chen, "Phase synchronization of coupled chaotic multiple time scales systems," Chaos, Solitons \& Fractals, vol. 20, no. 3, pp. 639-647, 2004.

[20] L. M. Tam, J. H. Chen, H. K. Chen, and W. M. Si Tou, "Generation of hyperchaos from the Chen-Lee system via sinusoidal perturbation," Chaos, Solitons \& Fractals, vol. 38, no. 3, pp. 826-839, 2008.

[21] K. Sebastian Sudheer and M. Sabir, "Modified function projective synchronization of hyperchaotic systems through OpenPlus-Closed-Loop coupling," Physics Letters A, vol. 374, no. 1920, pp. 2017-2023, 2010.

[22] M. Sun, L. Tian, and Q. Jia, "Adaptive control and synchronization of a four-dimensional energy resources system with unknown parameters," Chaos, Solitons \& Fractals, vol. 39, no. 4, pp. 1943-1949, 2009.

[23] Z. Li and X. Zhao, "Generalized function projective synchronization of two different hyperchaotic systems with unknown parameters," Nonlinear Analysis: Real World Applications, vol. 12, no. 5, pp. 2607-2615, 2011. 


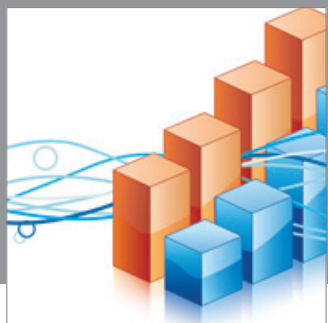

Advances in

Operations Research

mansans

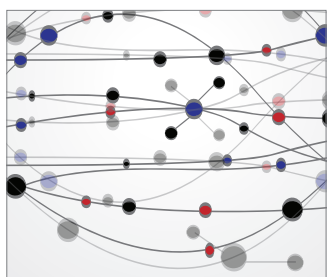

The Scientific World Journal
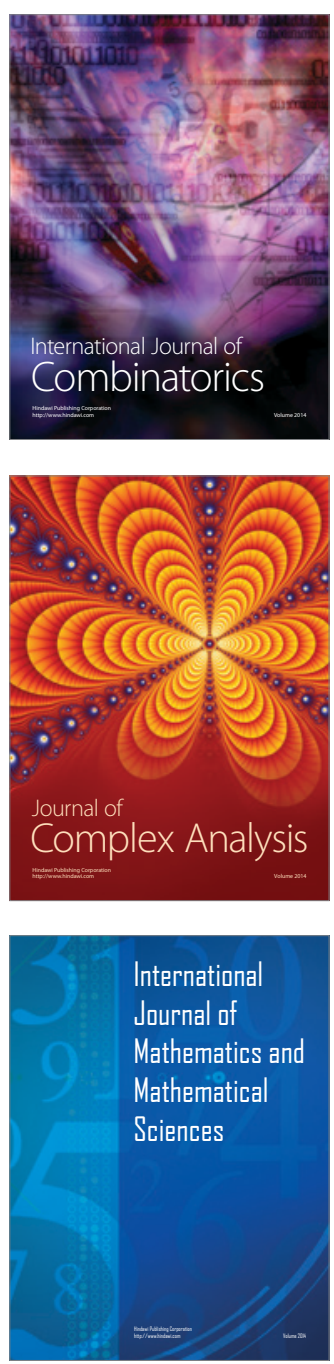
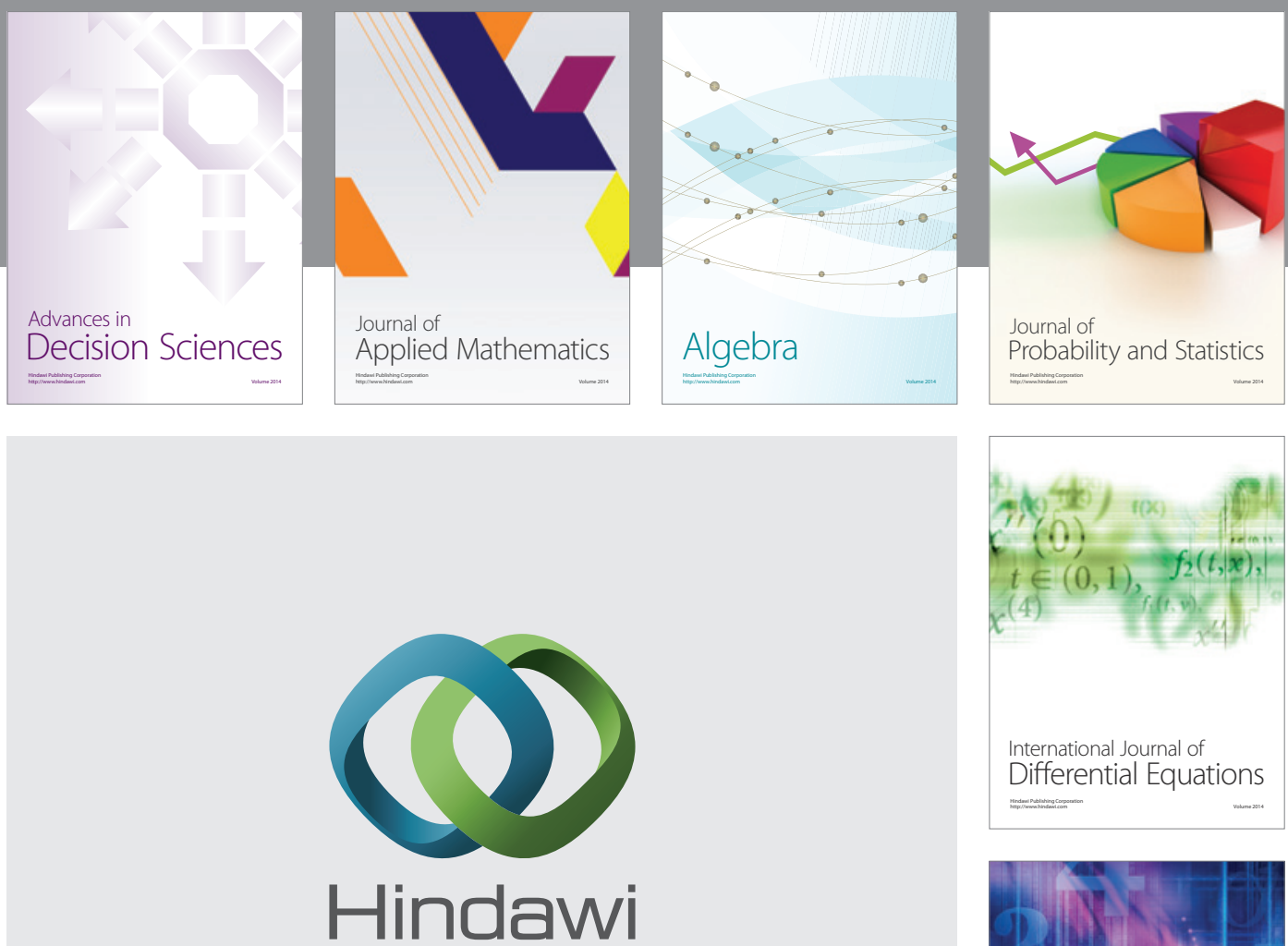

Submit your manuscripts at http://www.hindawi.com
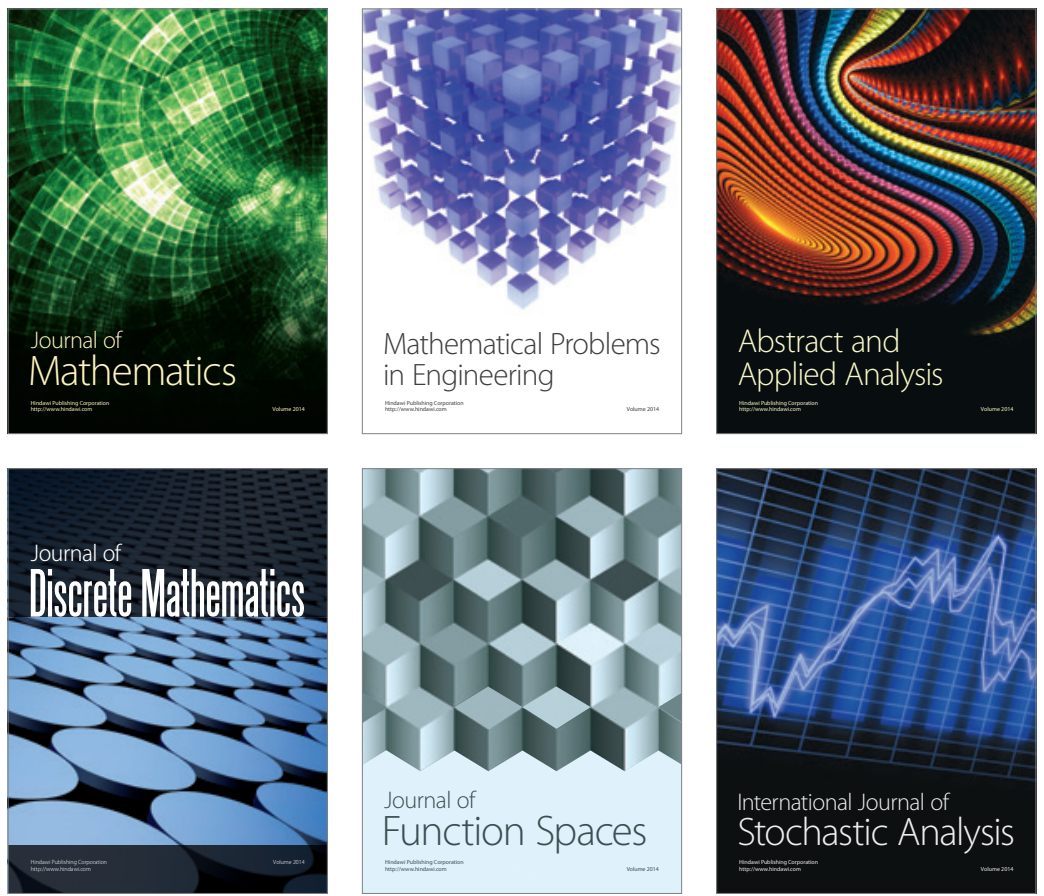

Journal of

Function Spaces

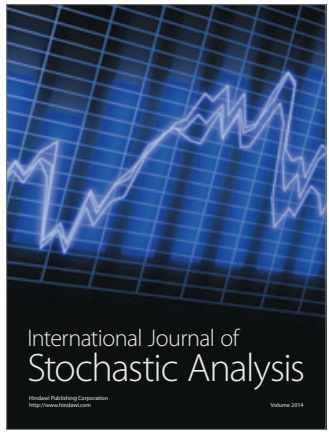

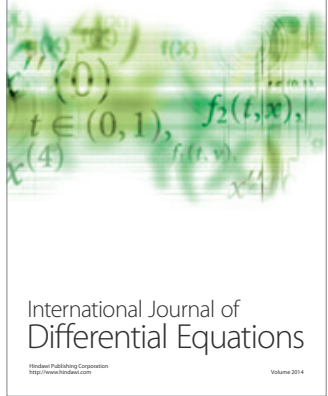
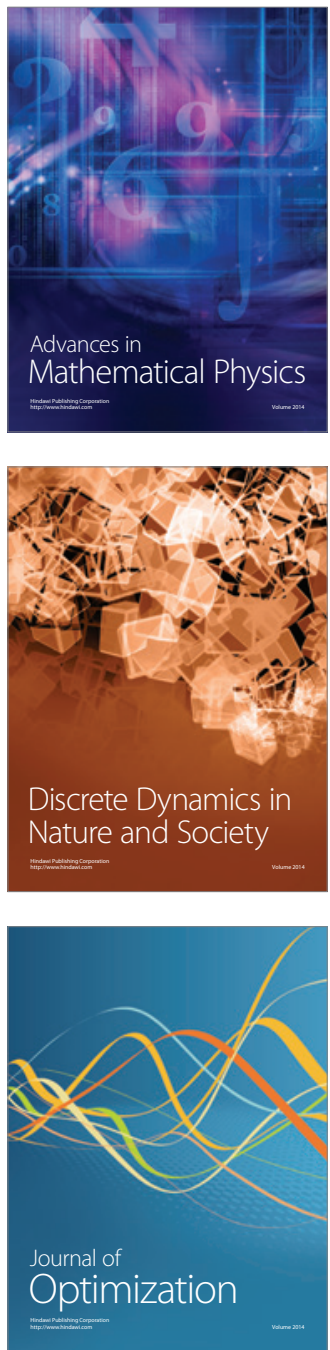